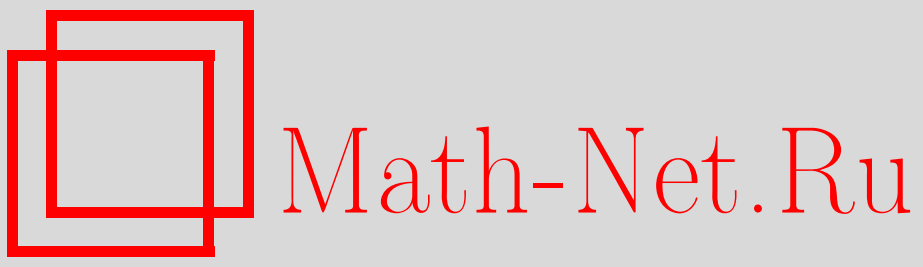

С. Ю. Новак, О распределении отношения сумм случайных величин, Теория вероятн. и ее примен., 1996, том 41, выпуск $3,533-560$

DOI: https://doi.org/10.4213/tvp3134

Использование Общероссийского математического портала Math-Net.Ru подразумевает, что вы прочитали и согласны с пользовательским соглашением http://www.mathnet.ru/rus/agreement

Параметры загрузки:

IP : 54.197 .130 .99

26 апреля 2023 г., 11:54:02

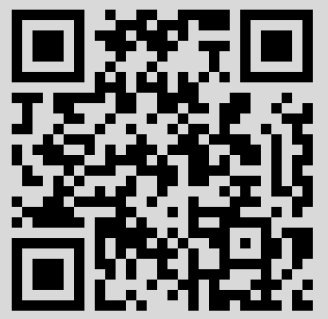


(C) 1996 г.

HOBAK C. Ю.*

\section{О РАСПРЕДЕЛЕНИИ ОТНОШЕНИЯ СУММ СЛУЧАЙНЫХ ВЕЛИЧИН}

В работе доказаны оценки типа Берри-Эссеена для распределения отношения $Z_{n}$ сумм случайных величй. Даются приложения полученных результатов к задачам непараметрического оценивания функции регрессии и индекса экстремального распределения в схемах последовательного оценивания.

Ключевые слова и бразы: оценки типа Берри-Эссеена, регрессия, ядерная оценка.

1. Введение. Пусть $(\xi, \eta),\left(\xi_{1}, \eta_{1}\right), \ldots$ - последовательность независимых пар случайных величин. Введем обозначения:

$$
\begin{aligned}
S_{n} & =\sum \xi_{i}, \quad T_{n}=\sum \eta_{i}, \quad Z_{n}=\frac{S_{n}}{T_{n}}, \quad \xi_{i}^{*}=\xi_{i}-\eta_{i} \frac{m_{1}}{m_{2}}, \quad S_{n}^{*}=\sum \xi_{i}^{*}, \\
m_{1} & \equiv m_{1}(n)=\sum \mathbf{E} \xi_{i}, \quad m_{2}=m_{2}(n)=\sum \mathbf{E} \eta_{i}, \\
\sigma_{1}^{2} & \equiv \sigma_{1}^{2}(n)=\sum \mathbf{D} \xi_{i}^{*}, \quad \sigma_{2}^{2} \equiv \sigma_{2}^{2}(n)=\sum \mathbf{D} \eta_{i}, \\
\mu_{1} & \equiv \mu_{1}(n)=\sum \mathbf{E}\left|\xi_{i}^{*}\right|^{3}, \quad \mu_{2}^{*} \equiv \mu_{2}^{*}(n)=\sum \mathbf{E}\left|\frac{\bar{\eta}_{i}}{\sigma_{2}}\right|^{3}, \\
\mu & \equiv \mu(n)=\mu_{1}+3 \mu_{2}^{*} \sigma_{1}^{3}+3 \sigma_{1}\left(\mu_{1}^{2} \mu_{2}^{*}\right)^{1 / 3}, \quad\|x\|_{*}^{3}=|x|^{3}+\left(\frac{2}{3}\right)^{3} .
\end{aligned}
$$

Символ $\sum$, если не оговорено противное, означает суммирование по множеству $J_{n}=\{1, \ldots, n\}$, черта нац случайной величиной (с.в.) означает центрирование математическим ожиданием.

Мы предполагаем, что $\xi_{i}=0$, как только $\eta_{i}=0$, а также что $m_{2} \neq 0$. Неопределенность вида $\frac{0}{0}$ считается равной нулю.

Асимптотические свойства распределения дроби $Z_{n}$ изучались Успенским [1], Кимболлом [2], Стояновым [3], Новаком и Утевым [4] и другими. Авторы вышеназванных статей основное внимание уделяли изучению асимптотики двух первых моментов $Z_{n}$ при $n \rightarrow \infty$ в предположении, что пары $\left\{\left(\xi_{i}, \eta_{i}\right)\right\}$ одинаково распределены. В частности, в [4] доказан следующий результат.

*Сибирская государственная геодезическая академия, ул. Плахотного, 10, 630108 Новосибирск, Россия. 
Теорема А. Если пары $\left\{\left(\xi_{i}, \eta_{i}\right)\right\}$ одинаково распределены, $\mathbf{E} \eta+$ $\mathbf{E}|\xi|<\infty u$

$$
\mathbf{P}\left\{\eta_{i} \geqslant 0\right\}=1 \quad(i \geqslant 1),
$$

mo сxодимость

$$
\mathbf{E} Z_{n}-\frac{\mathbf{E} \xi}{\mathbf{E} \eta} \longrightarrow 0 \quad(n \rightarrow \infty)
$$

имеет место тогда и только тогда, когда

$$
\mathbf{E}\left(\frac{\left|\xi_{1}\right|}{T_{m}}\right)<\infty \quad(\exists m \geqslant 1) .
$$

Если выполнено (1.3) и $\mathbf{E}|\xi| \eta^{2}+\mathbf{E} \eta^{4}<\infty$, то при $n \rightarrow \infty$

$$
\left|\mathbf{E} Z_{n}-\frac{\mathbf{E} \xi}{\mathbf{E} \eta}+\frac{\mathbf{E} \xi^{*} \eta}{n(\mathbf{E} \eta)^{2}}\right|=O\left(n^{-2}\right) .
$$

Если в дополнение $\mathbf{E} \xi^{2}(1+\eta)+\mathbf{E}\left(\xi_{1} / T_{m}\right)^{2}<\infty(\exists m \geqslant 2)$, то при $n \rightarrow \infty$

$$
\left|\mathbb{E}\left(Z_{n}-\frac{\mathbf{E} \xi}{\mathbf{E} \eta}\right)^{2}-\frac{\mathbf{D} \xi^{*}}{n(\mathbf{E} \eta)^{2}}\right|=O\left(n^{-2}\right) .
$$

Как отмечалось в [3], [4], задача о распределении с.в. $Z_{n}$ имеет интересные приложения к теории $n$-кратных интегралов. Классическая ядерная оценка функции регрессии также имеет вид отношения сумм случайных величин (см., например, [5], [6]). Другие примеры использования статистик вида $Z_{n}$ можно найти в $[7],[8]$.

Заметим, что случайная величина $Z_{n}$ асимптотически нормальна при условиях, обеспечивающих ЦПТ для $S_{n}^{*}$ и ЗБЧ для $T_{n}$.

$\mathrm{B}$ данной работе оценивается точность нормальной аппроксимации распределения случайных величин $Z_{n}$; уточняется и обобщается результат теоремы A.

Основные утверждения приведены в п. 2. Разделы 3-5 посвящены приложениям результатов п. 2 к ряду задач непараметрической статистики. Доказательства приводимых ниже теорем 2.1-2.5 отнесены в разделы 6,7 .

2. Формулировки основных результатов. Пусть $\varphi$ и $\Phi-$ плотность и функция распределения нормального $\mathcal{N}(0,1)$ закона; $C_{*}=$ 0.7915 и $C_{+}=31.995-$ констаңты в неравенствах Берри-Эссеена [11], [12]. Положим

$$
\Delta(n, x)=\left|\mathbf{P}\left\{S_{n}^{*}<\frac{x \sigma_{1} T_{n}}{m_{2}}\right\}-\Phi(x)\right| .
$$

Теорема 2.1. Для всякого $n \geqslant 1$

$$
\sup _{x \in \mathbf{R}} \Delta(n, x) \leqslant C_{*}\left(\frac{3}{2 \sigma_{1}}\right)^{3} \mu+\frac{8 \sigma_{2}}{e \sqrt{2 \pi}\left|m_{2}\right|}+17\left(\frac{\sigma_{2}}{m_{2}}\right)^{2} .
$$


Положим $B_{n}=\left\{x:\left|x \sigma_{2}\right| \leqslant\left|m_{2}\right| / 3\right\}$.

Теорема 2.2. Для всякого $n \geqslant 1$ при $x \in B_{n}$ выполняется неравенство

$$
\Delta(n, x) \leqslant C_{+}\|x\|_{*}^{-3} \mu \sigma_{1}^{-3}+x^{2} \varphi\left(\frac{x}{2}\right) \frac{\sigma_{2}}{\left|m_{2}\right|} .
$$

Отметим, что в теоремах 2.1, 2.2 не используется условие (1.1). Если (1.1) выполнено, то деление на $T_{n}$ сохраняет неравенство под знаком Р в (2.1), и соотношения (2.2)-(2.3) дают оценки скорости сходимости в предельной теореме для $Z_{n}$.

Рассмотрим вопрос об асимптотике первых двух моментов $Z_{n}$. Прежде всего, заметим, что условие (1.3) не обеспечивает, вообще говоря, существования $\mathrm{E} Z_{n}$ для всех достаточно больших $n$. Действительно, пусть $\eta, \eta_{1}, \eta_{2}, \ldots$ независимы и $\mathbf{P}\{\eta=1\}=\mathbf{P}\{\eta=-1\}=\frac{1}{2}, \xi_{i} \equiv 1$. Тогда среднее $\mathbf{E}\left(\sum \xi_{i} / \sum \eta_{i}\right) \equiv n \mathbf{E}\left(1 / \sum \eta_{i}\right)$ не определено для четных $n$, хотя условие (1.3) выполнено при любом нечетном $m$.

Чтобы гарантировать суцествование $\mathrm{E} Z_{n}$, мы будем предполагать в дальнейшем, что выполнено условие (1.1). Кроме того, будем считать, что для всякого $i \in J_{n}$ выбрана «окрестность» $A_{i} \in J_{n}$ «точки» $i$ так, что $\max _{i} \operatorname{card} A_{i} \leqslant k_{*}<\infty$.

Положим $A(i, j)=A_{i} \cup\{j\}$,

$$
\begin{gathered}
\eta_{(i)}=\sum_{j \in A_{i}} \eta_{j}, \\
T_{n i j}=T_{n}-\eta_{i}-\eta_{j} 1\{i \neq j\}, \quad \widehat{T}_{n i j}=\sum_{k \notin A(i, j)} \eta_{k}, \\
m^{+} \equiv m^{+}(n)=\min _{i, j \in J_{n}} \frac{\mathbf{E} \widehat{T}_{n i j}}{3}, \\
\rho_{1} \equiv \delta^{-3} m_{2}^{-2} \sum \mathbf{E}\left|\xi_{i}^{*}\right| \eta_{i}^{2}, \\
\rho_{2} \equiv 3 \delta^{-3} m_{2}^{-2} \sum\left|\mathbf{E} \xi_{i}^{*}\right| \mathbf{D} \eta_{i}+\delta^{-1} \alpha(n) \sum\left|\mathbf{E} \xi_{i}^{*}\right|, \\
\rho_{3} \equiv m_{2} \alpha(n) \sum \mathbf{E}\left|\frac{\xi_{i}^{*}}{\eta_{(i)}}\right|, \quad \rho_{4}=\rho^{*} \sum \frac{\left|\mathbf{E} \xi_{i}^{*} \bar{\eta}_{i}\right|}{m_{2}}, \quad \delta=\frac{m^{+}}{m_{2}}, \\
\rho^{*} \equiv 3 \alpha(n)+3\left(\frac{\sigma_{2}}{m^{+}}\right)^{2}, \quad \alpha(n) \equiv \exp \left\{\frac{-\left(m^{+}\right)^{2}}{8 \sum \mathbf{E} \eta_{i}^{2}}\right\} .
\end{gathered}
$$

Теорема 2.3. Для всякого $n \geqslant k_{*}$

$$
\left|m_{2} \mathbf{E} Z_{n}-m_{1}+\frac{\sum \mathbf{E} \xi_{i}^{*} \bar{\eta}_{i}}{m_{2}}\right| \leqslant \sum_{i=1}^{4} \rho_{i}(n) .
$$

Следствие 2.1. Если пары $\left\{\left(\xi_{i}, \eta_{i}\right)\right\}$ одинаково распределены $и$ $\mathbf{E}\left|\xi_{1}\right| \eta_{1}^{2}+\mathbf{E} \eta_{1}^{3}+\mathbf{E}\left|\xi_{1}\right| / T_{m}<\infty(\exists m>1)$, mo имеem место (1.4). 
Положим $\eta_{i j}=\sum_{k \in A_{i} \cup A_{j}} \eta_{k}$,

$$
\begin{aligned}
& R_{1} \equiv 6 \delta^{-4} m_{2}^{-2}\left(\sum \mathbf{E}\left|\bar{\xi}_{i}^{*} \bar{\eta}_{i}\right|\right)^{2}, \quad R_{2} \equiv 2 \delta^{-3} \sum \mathbf{E}\left(\bar{\xi}_{i}^{*}\right)^{2}\left|\frac{\bar{\eta}_{i}}{m_{2}}\right| \\
& R_{3} \equiv \rho^{*} \sigma_{1}^{2}, \quad R_{5} \equiv m_{2}^{2} \alpha(n)\left(\left(\sum_{i} \mathbf{E}\left|\frac{\xi_{i}^{*}}{\eta_{(i)}}\right|\right)^{2}+\sum_{i} \sum_{j}^{*} \mathbf{E}\left|\xi_{i}^{*} \xi_{j}^{*}\right| \eta_{i j}^{-2}\right) \\
& R_{4} \equiv \delta^{-2} \alpha(n) \sum_{j}\left|\mathbf{E} \xi_{j}^{*}\right| \sum_{i} \mathbf{E}\left|2 \xi_{i}^{*}-\mathbf{E} \xi_{i}^{*}\right|, \quad R_{8} \equiv 3 \delta^{-4} \sum \mathbf{E}\left(\frac{\bar{\xi}_{i}^{*} \bar{\eta}_{i}}{m_{2}}\right)^{2}, \\
& R_{6} \equiv 24 m_{2}^{-3} \delta^{-5}\left(\sum_{i} \mathbf{E}\left|\bar{\xi}_{i}^{*} \bar{\eta}_{i}\right|\right)\left(\sum_{j} \mathbf{E}\left|\bar{\xi}_{j}^{*}\left(\bar{\eta}_{j}\right)^{2}\right|\right), \\
& R_{7}^{*}=5 \alpha(n)+10 \delta^{-4}\left(\frac{\sigma_{2}}{m_{2}}\right)^{2}, \quad \rho_{1}^{*} \equiv 4 \alpha(n)+6 \delta^{-3}\left(\frac{\sigma_{2}}{m_{2}}\right)^{2} \\
& R_{7} \equiv 6 m_{2}^{-2}\left(\sum_{i}\left|\mathbf{E} \bar{\xi}_{i}^{*} \eta_{i}\right|\right)^{2} R_{7}^{*}+6 m_{2}^{-2} \sum\left(\mathbf{E} \bar{\xi}_{i}^{*} \eta_{i}\right)^{2}
\end{aligned}
$$

$$
\begin{aligned}
R_{9}(n, i) \equiv & 10 \alpha(n)+3 \mathbf{E}\left(\frac{\bar{\eta}_{i}}{m_{2}}\right)^{2}+4 \sum\left|\mathbf{E}\left(\frac{\bar{\eta}_{i}}{m_{2}}\right)^{3}\right| \\
& +5 \delta^{-6} m_{2}^{-4}\left\{3 \sigma_{2}^{4}+\sum \mathbf{E}\left(\bar{\eta}_{j}\right)^{4}\right\} \\
R_{9}= & \sum_{i} R_{9}(n, i) \mathbf{D} \xi_{i}^{*}, \quad R_{10}=2 \sum_{i} \mathbf{E}\left|\left(\bar{\xi}_{i}^{*}\right)^{2} \frac{\bar{\eta}_{i}}{m_{2}}\right| \rho_{1}^{*}
\end{aligned}
$$

где суммирование под знаком $\sum_{j}^{*}$ ведется по множеству $\left\{j: A_{j} \cap A_{i} \neq \varnothing\right\}$.

Теорема 2.4. Предположим, что саrd $A_{i} \geqslant 2\left(i \in J_{n}\right)$. При $n \geqslant k_{*}$

$$
\left|\mathrm{E}\left(m_{2} Z_{n}-m_{1}\right)^{2}-\sigma_{1}^{2}\right| \leqslant \sum_{j=1}^{5} R_{j}(n) .
$$

Следствие 2.2. Если пары $\left\{\left(\xi_{i}, \eta_{i}\right)\right\}$ одинаково распределены и $\mathbf{E}\left(\eta^{3}+|\xi| \eta^{2}\right)+\mathbf{E} \xi_{1}^{2}\left(1+\eta_{1}+\left(T_{m}\right)^{-2}\right)<\infty(\exists m \geqslant 2)$, mo uмeет место (1.5).

Теорема 2.5. В условиях теоремы 2.4

$$
\begin{aligned}
& \mid \mathbf{E}\left(m_{2} Z_{n}-m_{1}\right)^{2}-\sigma_{1}^{2}+2 \sum \frac{\mathbf{E}\left(\bar{\xi}_{i}^{*}\right)^{2} \bar{\eta}_{i}}{m_{2}}-3\left(\frac{\sigma_{1} \sigma_{2}}{m_{2}}\right)^{2} \\
& -6\left(\sum \frac{\mathbf{E} \xi_{i}^{*} \bar{\eta}_{i}}{m_{2}}\right)^{2} \mid \leqslant \sum_{j=5}^{10} R_{j}(n) .
\end{aligned}
$$

Следствие 2.3. Если пары $\left\{\left(\xi_{i}, \eta_{i}\right)\right\}$ одинаково распределены, удовлетворяют (1.1) и $\mathbf{E}\left(\xi_{1}^{2}\left(1+\eta_{1}^{2}\right)+\eta_{1}^{4}+\left(\xi_{1} / T_{m}\right)^{2}\right)<\infty$ для некоторого 
$m \geqslant 2$, mo npu $n \rightarrow \infty$

$$
\begin{gathered}
\mid n^{2} \mathbf{E}\left(Z_{n} \mathbf{E} \eta-\mathbf{E} \xi\right)^{2}-n \mathbf{D} \xi^{*}+2 \frac{\mathbf{E}\left(\xi^{*}\right)^{2} \bar{\eta}}{\mathbf{E} \eta} \\
-3 \frac{\left(\mathbf{D} \xi^{*}\right) \mathbf{D} \eta}{(\mathbf{E} \eta)^{2}}-6 \frac{\left(\mathbf{E} \xi^{*} \eta\right)^{2}}{(\mathbf{E} \eta)^{2}} \mid=O\left(n^{-1}\right)
\end{gathered}
$$

II р и м е р 2.1. Пусть $\xi_{1}, \ldots, \xi_{n}$ - независимые случайные величины с экспоненциальным распределением $\mathrm{E}(b)$. Естественной оценкой числа $b$ является статистика

$$
b_{n}=\frac{n}{\sum \xi_{i}}
$$

Из теоремы 2.2 следует, что при $3|x| \leqslant \sqrt{n}$

$$
\begin{aligned}
\Delta_{*} & \equiv\left|\mathbf{P}\left\{\sqrt{n}\left(1-\frac{b_{n}}{b}\right)<x\right\}-\Phi(x)\right| \\
& \leqslant \frac{7 C_{+}\|x\|_{*}^{-3}\left(22 e^{-1}-4\right)+x^{2} \varphi(x / 2)}{\sqrt{n}} .
\end{aligned}
$$

Отметим, что $\mathrm{E}\left(\xi_{1}+\xi_{2}+\xi_{3}\right)^{-2}<\infty$.

3. Оценивание функции регрессии. Пусть $\left(X_{1}, Y_{1}\right), \ldots$, $\left(X_{n}, Y_{n}\right)$ - выборка независимых наблюдений над распределением пары $(X, Y)$ со значениями из $\mathbf{R}^{d} \times \mathbf{R}$, и пусть $\mathbf{E}|Y|<\infty$. Требуется оценить функцию регрессии

$$
\psi(x)=\mathbf{E}\{Y \mid X=x\} .
$$

Будем предполагать, что распределение случайного вектора $X$ имеет плотность $f$ относительно меры Лебега в $\mathbf{R}^{d}$. В дальнейшем в этом разделе символ $f_{\gamma}$ обозначает плотность распределения с.в. $\gamma$.

Классическая (см. [5], [6]) оценка функции $\psi(x)$ имеет вид

$$
\widehat{\psi}_{n}(x)=\frac{\sum Y_{i} f_{\gamma}\left(\varepsilon^{-1}\left(X_{i}-x\right)\right)}{\sum f_{\gamma}\left(\varepsilon^{-1}\left(X_{i}-x\right)\right)},
$$

где $\gamma$ - случайный вектор со значениями в $\mathbf{R}^{d}$, выбираемый статистиком,

$$
0<\varepsilon \equiv \varepsilon(n) \longrightarrow 0, \quad n \varepsilon^{d} \rightarrow \infty \quad(n \rightarrow \infty) .
$$

Отметим, что оценка (3.1) может быть переписана в виде

$$
\widehat{\psi}_{n}(x)=\frac{\sum Y_{i} f_{x+\varepsilon \gamma}\left(X_{i}\right)}{\sum f_{x+\varepsilon \gamma}\left(X_{i}\right)} .
$$

Последняя форма записи представляется нам более удобной. 
Хорошо известно, что оценка $\widehat{\psi}_{n}(x)$ состоятельна при выполнении условия (3.2) и определенных предположениях на $\gamma$, а при дополнительных ограничениях имеет место сходимость

$$
\left(\widehat{\psi}_{n}(x)-\psi(x)\right) \kappa^{-1}(x) \sqrt{n \varepsilon^{d}} \Longrightarrow \mathcal{N}(0,1) \quad(n \rightarrow \infty)
$$

где

$$
\begin{aligned}
\kappa^{2}(x) & =f^{-1}(x) \lambda(x) \mathbf{E} f_{\gamma}(\gamma), \\
\lambda(\cdot) & =\mathbf{E}\left\{(Y-\psi(x))^{2} \mid X=\cdot\right\} .
\end{aligned}
$$

При построении доверительных интервалов для $\psi(x)$ важным представляется вопрос о точности нормальной аппроксимации распределения статистики $\psi_{n}$. Цель данного раздела - оценить скорость сходимости в предельных теоремах для оценок функции регрессии в схемах последовательного оценивания.

Имеется тесная связь между оценками плотности распределения и оценками регрессии. Так, (3.1) есть аналог введенной Розенблатом [19] ядерной оценки плотности. Следующая оценка $\psi_{n}(x)$ соответствует оценке плотности, предложенной Дехойвельсом [9] (числа $\left\{\varepsilon_{i}\right\}$ и функция $H$ выбираются статистиком):

$$
\psi_{n}=\frac{\sum H\left(\varepsilon_{i}\right) Y_{i} f_{x+\varepsilon_{i} \gamma}\left(X_{i}\right)}{\sum H\left(\varepsilon_{i}\right) f_{x+\varepsilon_{i} \gamma}\left(X_{i}\right)} .
$$

Частным случаем (3.4) является статистика

$$
\psi_{n}^{*}=\frac{\sum Y_{i} f_{x+\varepsilon_{i} \gamma}\left(X_{i}\right)}{\sum f_{x+\varepsilon_{i} \gamma}\left(X_{i}\right)} .
$$

Отметим, что (3.5) есть аналог оценки плотности, предложенной Волвертоном и Вагнером [10].

Положим $\nu(\cdot)=\mathbf{E}\left\{|Y-\psi(x)|^{3} \mid X=\cdot\right\}$,

$$
H_{n k}=\sum H^{k+1}\left(\varepsilon_{i}\right) \varepsilon_{i}^{-k d}, \quad H_{n} \equiv H_{n 0}, \quad H_{n k t}=\sum H^{k+1}\left(\varepsilon_{i}\right) \varepsilon_{i}^{t-k d} .
$$

В дальнейшем символ $|\cdot|$, примененный к вектору или матрице, означает операторную норму; $\|g\|=\sup _{x}|g(x)|$. Числа $r_{i} \equiv r_{i}(x), i=1,2,3,4$ определим равенствами

$$
\begin{aligned}
r_{1}(x) & =\left(\frac{\mathbf{E} f_{\gamma}(\gamma)}{f(x)}\right)^{1 / 2}\left(\frac{3}{\sqrt{2 \pi}}+20 \mathbf{E}\left(\frac{f_{\gamma}(\gamma)}{\mathbf{E} f_{\gamma}(\gamma)}\right)^{2} \frac{\nu(x)}{\lambda^{3 / 2}(x)}\right), \\
2 \sqrt{\pi} r_{2}(x) & =\left(\left\|(f \psi)^{\prime \prime}\right\|+\|\psi\|\left\|f^{\prime \prime}\right\|\right) \frac{\mathbf{E}|\gamma|^{2}}{\kappa(x) f(x)}, \\
\sqrt{2 \pi} r_{3}(x) & =\frac{\left\|(\lambda f)^{\prime \prime}\right\| \mathbf{E} f_{\gamma}(\gamma)|\gamma|^{2}}{\lambda(x) f(x) \mathbf{E} f_{\gamma}(\gamma)}, \quad \sqrt{2 \pi} r_{4}(x)=\left\|f^{\prime \prime}\right\| f^{-1}(x) \mathbf{E}|\gamma|^{2} .
\end{aligned}
$$


Будем счита́ть, что распределение вектора $\gamma$ симметрично и выполнены следуюшие условия: $\varepsilon_{i} \rightarrow 0$ при $i \rightarrow \infty$,

$$
\begin{gathered}
\mathbf{E}\left(1+f_{\gamma}^{2}(\gamma)\right)\left(1+|\gamma|^{2}\right)<\infty, \\
\frac{H_{n 1}}{H_{n}^{2}} \rightarrow 0, \quad \frac{H_{n 02}^{2} H_{n 1}}{H_{n 12} H_{n}^{2}} \longrightarrow 0, \quad H_{n k} \rightarrow \infty \quad(n \rightarrow \infty, k=0,1,2) .
\end{gathered}
$$

Кроме того, будем предполагать, что при $i=0,1,2$

$$
\left\|f^{(i)}\right\|+\left\|\lambda^{(i)}\right\|+\left\|\psi^{(i)}\right\|+\left\|\nu^{(i)}\right\|<\infty .
$$

Теорема 3.1. При всех достаточно больиих $n$

$$
\begin{aligned}
& \sup _{y}\left|\mathbf{P}\left\{\psi_{n}(x)-\psi(x)<y \kappa(x) H_{n 1}^{1 / 2} H_{n}^{-1}\right\}-\Phi(y)\right| \\
& \quad \leqslant r_{1}(x) \frac{H_{n 2}}{H_{n 1}^{3 / 2}}+r_{2}(x) H_{n 02} H_{n 1}^{-1 / 2}+r_{3}(x) \frac{H_{n 12}}{H_{n 1}}+r_{4}(x) \frac{H_{n 02}}{H_{n}}
\end{aligned}
$$

где $\Phi$ - функция распределения нормальной $\mathcal{N}(0,1)$ случайной величиHbl.

Следствие 3.1. Eсли $\varepsilon_{i} \equiv \varepsilon_{n}, H\left(\varepsilon_{n}\right) \equiv 1 u \varepsilon_{n} \rightarrow 0, n \varepsilon_{n}^{d} \rightarrow \infty$, mo при всех достаточно больиих $n$

$$
\begin{aligned}
& \sup _{y}\left|\mathbf{P}\left\{\psi_{n}(x)-\psi(x)<y \kappa(x)\left(n \varepsilon_{n}^{d}\right)^{-1 / 2}\right\}-\Phi(y)\right| \\
& \quad \leqslant r_{1}\left(n \varepsilon_{n}^{d}\right)^{-1 / 2}+r_{2} \varepsilon_{n}^{2}\left(n \varepsilon_{n}^{d}\right)^{1 / 2}+\left(r_{3}+r_{4}\right) \varepsilon_{n}^{2} \\
& \sim r_{1}\left(n \varepsilon_{n}^{d}\right)^{-1 / 2}+r_{2} \varepsilon_{n}^{2}\left(n \varepsilon_{n}^{d}\right)^{1 / 2}
\end{aligned}
$$

Отметим, что правая часть соотношения (3.8) достигает минимума при $\varepsilon \equiv \varepsilon_{n}=\left(d r_{1} /\left((4+d) r_{2} n\right)\right)^{1 /(2+d)}$. При этом скорость сходимости в (3.8) есть $O\left(n^{-1 /(2+d)}\right)$.

Положим $r_{i} \equiv r_{i}(x)=r_{i}^{*}(x) /(\kappa(x) \sqrt{\pi})(i=5,6)$, где

$$
\begin{aligned}
& r_{5}^{*}=\left(\|\psi\|\left\|f^{(4)}\right\|+\left\|(\psi f)^{(4)}\right\|\right) \frac{\mathbf{E}|\gamma|^{4}}{24 f(x)}, \\
& r_{6}^{*}=\left\|f^{\prime \prime}\right\|\left(\left\|(\psi f)^{\prime \prime}\right\|+\|\psi\|\left\|f^{\prime \prime}\right\|\right)\left(\frac{\mathbf{E}|\gamma|^{2}}{2 f(x)}\right)^{2} .
\end{aligned}
$$

Определим функции $w, w^{*}$ равенствами

$$
w(\cdot)=\frac{\mathbf{E}\left((\psi f)^{\prime \prime}-\psi f^{\prime \prime}\right) \gamma \gamma}{2 f}, \quad w^{*}(x) \equiv w^{*}(x, n)=w(x) \frac{H_{n 02}}{H_{n}} .
$$

Теорема 3.2. Пусть $\left\|f^{(4)}\right\|+\left\|\psi^{(4)}\right\|<\infty$. Тогда при всех достаточно больиих $n$

$$
\sup _{y}\left|\mathbf{P}\left\{\psi_{n}(x)-\psi(x)<y \kappa(x) H_{n 1}^{1 / 2} H_{n}^{-1}\right\}-\Phi\left(y-\frac{w(x)}{\kappa(x)} H_{n 02} H_{n 1}^{-1 / 2}\right)\right|
$$




$$
\leqslant r_{1} \frac{H_{n 2}}{H_{n 1}^{3 / 2}}+r_{3} \frac{H_{n 12}}{H_{n 1}}+r_{4} \frac{H_{n 02}}{H_{n}}+r_{5} \frac{H_{n 04}}{H_{n 1}^{1 / 2}}+r_{6} \frac{H_{n 02}^{2}}{H_{n} H_{n 1}^{1 / 2}}
$$

Следствие 3.2. $E с л и\left\|f^{(4)}\right\|+\left\|\psi^{(4)}\right\|<\infty, \varepsilon_{i} \equiv \varepsilon_{n}, H\left(\varepsilon_{n}\right) \equiv 1, \varepsilon_{n} \rightarrow 0$, $n \varepsilon_{n}^{d} \rightarrow \infty$, то при всех достаточно больших $n$

$$
\begin{aligned}
& \sup _{y}\left|\mathbf{P}\left\{\psi_{n}(x)-\psi(x)<y \kappa(x)\left(n \varepsilon_{n}^{d}\right)^{-1 / 2}\right\}-\Phi\left(y-\frac{w(x)}{\kappa(x)}\left(n \varepsilon_{n}^{d+4}\right)^{1 / 2}\right)\right| \\
& \quad \leqslant r_{1}\left(n \varepsilon_{n}^{d}\right)^{-1 / 2}+\left(r_{3}+r_{4}\right) \varepsilon_{n}^{2}+\left(r_{5}+r_{6}\right) \varepsilon_{n}^{4}\left(n \varepsilon_{n}^{d}\right)^{-1 / 2} .
\end{aligned}
$$

Правая часть этого выражения достигает минимума при

$$
\varepsilon \equiv \varepsilon_{n}=n^{-1 /(d+4)}\left(\frac{-2 q_{2}+\sqrt{4 q_{2}^{2}+q_{1} q_{3} d(d+8)}}{q_{3}(d+8)}\right)^{2 /(4+d)},
$$

где $q_{1}=r_{1}(x), q_{2}=r_{3}(x)+r_{4}(x), q_{3}=r_{5}(x)+r_{6}(x)$; при этом порядок убывания правой части $\left(3.8^{*}\right)$ есть $n^{-2 /(d+4)}$.

До к а за т ель с т в о т е о ре м ы 3.1. Положим

$$
\eta_{i}=f_{x+\varepsilon_{i} \gamma}\left(X_{i}\right) H\left(\varepsilon_{i}\right), \quad \xi_{i}=Y_{i} \eta_{i} .
$$

Отметим, что для всякой измеримой функции $h: \mathbf{R} \rightarrow \mathbf{R}$

$$
\mathbf{E} h(Y) f_{x+\varepsilon \gamma}^{t+1}(X)=\varepsilon^{-t d} \mathbf{E} \widehat{H}(x+\varepsilon \gamma) f(x+\varepsilon \gamma) f_{\gamma}^{t}(\gamma) \quad(t \geqslant 0),
$$

где $\widehat{H}(y) \equiv \mathbf{E}\{h(Y) \mid X=y\}$. Нетрудно убедиться, что

$$
\begin{aligned}
\left|m_{1}-\psi(x) f(x) H_{n}\right| \leqslant & H_{n 02} \mathbf{E}|\gamma|^{2} \frac{\left\|(\psi f)^{\prime \prime}\right\|}{2} \\
\left|m_{2}-f(x) H_{n}\right| \leqslant & H_{n 02} \mathbf{E}|\gamma|^{2} \frac{\left\|f^{\prime \prime}\right\|}{2} \\
\left|\frac{m_{1}}{m_{2}}-\psi(x)\right| \leqslant & r_{n x}\left\|(\psi f)^{\prime \prime}\right\|+r_{n x}\left\|f^{\prime \prime}\right\|\left(\|\psi\|+r_{n x}\left\|(\psi f)^{\prime \prime}\right\|\right) \\
& \times\left(1-r_{n x}\left\|f^{\prime \prime}\right\|\right)^{-1} \sim r_{n x}\left(\left\|(\psi f)^{\prime \prime}\right\|+\left\|f^{\prime \prime}\right\|\|\psi\|\right)
\end{aligned}
$$

где $r_{n x}=f^{-1}(x) \mathbf{E}|\gamma|^{2} H_{n 02} /\left(2 H_{n}\right.$ ) (последнее неравенство справедливо при всех $n$, для которых $\left.r_{n x}<1\right)$. Вследствие (3.9)

$$
\mathbf{E}(Y-\psi(x))^{2} f_{x+\varepsilon \gamma}^{2}(X)=\varepsilon^{-d} \mathbf{E} \lambda(x+\varepsilon \gamma) f(x+\varepsilon \gamma) f_{\gamma}(\gamma) .
$$

Поэтому при $n \rightarrow \infty$

$$
\left|\sigma_{1}^{2}-\lambda(x) f(x) \mathbf{E} f_{\gamma}(\gamma) H_{n 1}\right| \leqslant\left\|(\lambda f)^{\prime \prime}\right\| \mathbf{E}|\gamma|^{2} f_{\gamma}(\gamma) H_{n 12}(1+o(1))
$$


(нетрудно выписать и явную оценку, однако это слишком загромоздило бы изложение). Далее,

$$
\begin{aligned}
\frac{\sigma_{1}}{m_{2}} & =\kappa(x) H_{n 1}^{1 / 2} H_{n}^{-1} \rho \\
|\rho-1| & \leqslant \sqrt{2 \pi}\left(r_{3}(x) H_{n 12} H_{n 1}^{-1}+r_{4}(x) H_{n 02} H_{n}^{-1}\right)
\end{aligned}
$$

при всех достаточно больших $n$. Отметим также, что при $n \rightarrow \infty$

$$
\frac{\sigma_{2}}{m_{2}} \sim\left(\frac{\mathrm{E} f_{\gamma}(\gamma)}{f(x)}\right)^{1 / 2} H_{n 1}^{1 / 2} H_{n}^{-1}
$$

Легко убедиться, что $\mu \leqslant 3 \mu_{1}+4 \sigma_{1}^{3} \mu_{2}^{*}$. Поэтому при $n \rightarrow \infty$

$$
\frac{\mu}{\sigma_{1}^{3}} \leqslant 7\left(\frac{\mathbf{E} f_{\gamma}(\gamma)}{f(x)}\right)^{1 / 2} \mathbf{E}\left(\frac{f_{\gamma}(\gamma)}{\mathbf{E} f_{\gamma}(\gamma)}\right)^{2} \frac{\nu(x)}{\lambda^{3 / 2}(x)} H_{n 2} H_{n 1}^{-3 / 2}(1+o(1)) .
$$

Из теоремы 2.1 с учетом неравенства $\left(H_{n 1}\right)^{2} \leqslant H_{n 2} H_{n}$ выводим:

$$
\sup _{u}\left|\mathbf{P}\left\{\psi_{n}(x)-\frac{m_{1}}{m_{2}}<\frac{u \sigma_{1}}{m_{2}}\right\}-\Phi(u)\right| \leqslant r_{1} H_{n 2} H_{n 1}^{-3 / 2}
$$

при всех достаточно больших $n$. Делая замену переменной $z=u+$ $\left(m_{1} / m_{2}-\psi(x)\right) m_{2} / \sigma_{1}$, получим:

$$
\sup _{z}\left|\mathbf{P}\left\{\psi_{n}(x)-\psi(x)<\frac{z \sigma_{1}}{m_{2}}\right\}-\Phi(z)\right| \leqslant r_{1} H_{n 2} H_{n 1}^{-3 / 2}+r_{2} H_{n 02} H_{n 1}^{-1 / 2}
$$

Положим $z=y / \rho$. Из $(3.10)-(3.13)$ и равенства

$$
\sup _{z} z \exp \left\{-\frac{z^{2}}{2}\right\}=\frac{1}{\sqrt{e}}
$$

вытекает утверждение теоремы 3.1 .

3 а м е ч а н и е. Если носитель распределения случайного вектора $\gamma$ ограничен, то (3.7) останется в силе, если в определении $r_{i}$ вместо участвующего в определении нормы $\|\cdot\|$ супремума по $\mathbf{R}^{d}$ взять супремум по произвольному фиксированному шару с центром в точке $x$. Если же вторые производные функций $f, \psi, \lambda, \nu$ негрерывны в окрестности $x$, то нормы можно заменить модулями значений в точке $x$ стоящих под знаками нормы функций.

Д ок аз а т ель ст в о т е о ре мы 3.2. Положим

$$
r_{7}(x, n)=r_{5}^{*} \frac{H_{n 04}}{H_{n}}+r_{6}^{*}\left(\frac{H_{n 02}}{H_{n}}\right)^{2} .
$$


Нетрудно проверить, что

$$
\left|\frac{m_{1}}{m_{2}}-\psi(x)-w^{*}(x)\right| \leqslant r_{7}(x, n)(1+o(1)) .
$$

Делая в (3.12) замену переменной $z=u+\left(m_{1} / m_{2}-\psi(x)\right) m_{2} / \sigma_{1}$, с учетом (3.11) получаем, что при $n \rightarrow \infty$

$$
\begin{aligned}
\sup _{z}\left|\mathbf{P}\left\{\psi_{n}(x)-\psi(x)<\frac{z \sigma_{1}}{m_{2}}\right\}-\Phi\left(z-w^{*}(x) \frac{m_{2}}{\sigma_{1}}\right)\right| \\
\leqslant r_{1} \frac{H_{n 2}}{H_{n 1}^{3 / 2}}+r_{7}(x, n) \frac{m_{2}}{\sigma_{1} \sqrt{2 \pi}}(1+o(1)) \\
\quad=r_{1} H_{n 2} H_{n 1}^{-3 / 2}+\left(r_{5}^{*} H_{n 04}+r_{6}^{*} H_{n 02}^{2} H_{n}^{-1}\right) \frac{H_{n 1}^{-1 / 2}}{\kappa(x) \sqrt{2 \pi}}(1+o(1)) .
\end{aligned}
$$

Заметим, что

$$
w^{*}(x) \frac{m_{2}}{\sigma_{1}}=w(x) \frac{H_{n 02}}{\kappa(x) H_{n 1}^{1 / 2} \rho} .
$$

Поэтому, принимая во внимание (3.14),

$$
\begin{aligned}
& \sup _{y}\left|\mathbf{P}\left\{\psi_{n}(x)-\psi(x)<y \kappa(x) H_{n 1}^{1 / 2} H_{n}^{-1}\right\}-\Phi\left(y-\frac{w(x)}{\kappa(x)} H_{n 02} H_{n 1}^{-1 / 2}\right)\right| \\
& \leqslant r_{1} H_{n 2} H_{n 1}^{-3 / 2}+\left(r_{5}^{*} H_{n 04}+r_{6}^{*} H_{n 02}^{2} H_{n}^{-1}\right) H_{n 1}^{-1 / 2} \frac{(1+o(1))}{\kappa(x) \sqrt{2 \pi}} \\
&+\frac{(1+o(1))}{\sqrt{2 \pi e}}|1-\rho|,
\end{aligned}
$$

что влечет за собой утверждение теоремы 3.2 .

4. Статистическое оценивание показателя скорости убывания «хвоста» неизвестного вероятностного распределения. Пусть $X_{1}, \ldots, X_{n}$ - независимые копии случайной величины $X$, pacnределение которой допускает при $x>0$ представление

$$
p(x) \equiv \mathbf{P}\{X \geqslant x\}=L(x) x^{-b} \quad(b>0),
$$

где функция $L(x)$ медленно меняется при $x \rightarrow \infty$, т.е.

$$
\lim _{x \rightarrow \infty} \frac{L(x t)}{L(x)}=1
$$

для любого $t>0$. Требуется оценить число $b$ по наблюдениям $X_{1}, \ldots, X_{n}$.

Различные оценки числа $b$ изучались в [21]-[36]. Хилл [21] предложил оценку

$$
\widehat{b}_{n}=\frac{N_{n}}{\sum_{i=1}^{N_{n}} \ln \left(X_{(n-i+1)} / X_{\left(n-N_{n}\right)}\right)},
$$


где $X_{(1)} \leqslant \cdots \leqslant X_{(n)}$ 一 вариационный ряд выборки $X_{1}, \ldots, X_{n}$, числа $\left\{N_{n}\right\}$ выбираются статистиком (здесь и ниже $\ln x=-\infty$, если $x \leqslant 0$ ). Распределение оценки

$$
b_{n}^{*}=\frac{\sum_{1}^{n} 1\left\{X_{i}>N_{n}\right\}}{\sum_{1}^{n} \ln \left(X_{i} / N_{n}\right) 1\left\{X_{i}>N_{n}\right\}},
$$

(числа $\left\{N_{n}\right\}$ выбираются статистиком) изучалось в [4], [28], где установлена следующая

Теорема В. Пусть последовательность чисел $\left\{N_{n}\right\}$ такова, что $n p\left(N_{n}\right) \rightarrow \infty u \gamma_{n}=\mathbf{E}\left\{\ln \left(X / N_{n}\right) \mid X>N_{n}\right\}-1 / b \longrightarrow 0$ npu $n \rightarrow \infty$. Toадa $b_{n}^{*} \stackrel{\mathbf{P}}{\longrightarrow} b$, причем сxодимость

$$
\left(n p\left(x_{n}\right)\right)^{1 / 2}\left(\frac{b_{n}^{*}}{b}-1\right) \Longrightarrow \mathcal{N}(0,1)
$$

имеет место в том и только том случае, если $n p\left(N_{n}\right) \gamma_{n}^{2} \longrightarrow 0$ при $n \rightarrow \infty$.

В частном случае $L(x)=a^{b} 1\{x \geqslant a\}, N_{n} \equiv a$, статистика $b_{n}^{*}$ совпадает с оценкой максимального правдоподобия.

Отметим, что теоремы о слабой сходимости во всех упомянутых работах имеют вид

$$
k_{n}\left(b_{n}-b\right) \Longrightarrow \mathcal{N}(0,1)
$$

где $k_{n} \rightarrow \infty$ при $n \rightarrow \infty$.

В настояшем разделе рассматривается оценка

$$
b_{n}=\frac{\sum Y_{0, i}}{\sum Y_{1, i}}
$$

где

$$
Y_{k, i}=\ln ^{k}\left(\frac{X_{i}}{N_{i, n}}\right) \mathbf{1}\left\{X_{i}>N_{i, n}\right\}
$$

числа $\left\{N_{i, n}\right\}$ выбираются статистиком. Оценка (4.6) является естественным обобщением статистики (4.3) для схем последовательного оценивания.

Принцип построения оценок (4.3), (4.6) становится понятным в свете следуюшего обстоятельства: условное распределение с.в. $\ln (X / N)$ при условии $X>N$ сходится при $N \rightarrow \infty$ к экспоненциальному закону $\mathrm{E}(b)$. Более того, при $N \rightarrow \infty$

$$
b^{k} \mathbf{E}\left\{\ln ^{k}\left(\frac{X}{N}\right) \mid X \geqslant N\right\}=\int_{0}^{\infty} L^{-1}(N) L\left(N e^{u / b}\right) e^{-u} d u^{k}=k !\left(1+v_{k}(N)\right),
$$

где для любого $\dot{k} \in \mathbf{N}$ при $N \rightarrow \infty$

$$
\begin{aligned}
& v_{k}(N) \equiv \frac{\int_{0}^{\infty} h_{N}(u) e^{-u} d u^{k}}{k !} \longrightarrow 0, \\
& h_{N}(u) \equiv h_{N}(u, b)=L^{-1}(N) L\left(N e^{u / b}\right)-1
\end{aligned}
$$


(справедливость (4.8) вытекает из свойств медленно меняюшихся функций [39]).

Естественно поставить вопрос о скорости сходимости в теоремах вида (4.5). Ниже мы выводим оценки типа Берри-Эссеена в предельных теоремах для распределений статистик $b_{n}$.

Положим $N_{i, n} \equiv N_{n, n}$ при $i>n$,

$$
\begin{aligned}
& p_{i}=p\left(N_{i, n}\right), \quad v_{k, i}=v_{k}\left(N_{i, n}\right), \quad r_{n}=\sum_{1}^{n} p_{i}, \\
& \varepsilon_{n}=r_{n}^{-1} \sum_{1}^{n} p_{i} v_{1, i}, \quad \delta_{n}=r_{n}^{-1} \sum_{1}^{n} p_{i} v_{2, i} .
\end{aligned}
$$

В этом разделе мы предполагаем, что

$$
\lim _{n \rightarrow \infty} r_{n}=\infty, \quad \lim _{\substack{n \rightarrow \infty \\ i \rightarrow \infty}} N_{i, n}=\infty .
$$

С помощью рассуждений, аналогичных приведенным в [28], легко убедиться, что условие (А) обеспечивает состоятельность оценки $b_{n}$. Применение теорем 2.1-2.5 позволяет получить следующие результаты.

Утверждение 4.1. При всех достаточно больших $n$

$$
\begin{gathered}
\sup _{y}\left|\mathbf{P}\left\{r_{n}^{1 / 2}\left(\frac{b_{n}}{b}-\frac{1}{1+\varepsilon_{n}}\right)<y\right\}-\Phi(y)\right| \\
\leqslant 80 r_{n}^{-1 / 2}+36 r_{n}^{-1}+\left|\delta_{n}-3 \varepsilon_{n}\right| \sqrt{\frac{2}{\pi}} .
\end{gathered}
$$

Утверждение 4.2. При всех достаточно больших $n$

$$
\begin{aligned}
& \sup _{20 y^{2}<r_{n}}\left|\mathbf{P}\left\{r_{n}^{1 / 2}\left(\frac{b_{n}}{b}-\frac{1}{1+\varepsilon_{n}}\right)<y\right\}-\Phi(y)\right| \\
& \quad \leqslant 2|y| \varphi\left(\frac{y}{2}\right)\left|\delta_{n}-3 \varepsilon_{n}\right|+\frac{27 C_{+} r_{n}^{-1 / 2}}{|y|^{3}+\frac{1}{4}}+2 y^{2} \varphi\left(\frac{y}{2}\right) r_{n}^{-1 / 2}
\end{aligned}
$$

Положим

$$
\widetilde{m}_{*} \equiv \widetilde{m}_{*}(n)=r_{n}^{-1} \sum_{1}^{n} p_{i}^{2}\left(\varepsilon_{n}-v_{1, i}\right), \quad \widetilde{m}^{\prime} \equiv \widetilde{m}^{\prime}(n)=r_{n}^{-1} \sum_{1}^{n} p_{i}^{2}\left(v_{1, i}-\varepsilon_{n}\right)^{2} .
$$

Запись $a_{n} \asymp b_{n}$ означает, что

$$
0<\liminf \frac{a_{n}}{b_{n}} \leqslant \limsup \frac{a_{n}}{b_{n}}<\infty .
$$

Ниже в этом разделе предполагается, что

$$
\min _{i, j \in J_{n}} \sum_{k \in J_{n} \backslash\{i, j\}} p_{k} \asymp r_{n} .
$$


Утверждение 4.3. При $n \rightarrow \infty$

$$
\left(b \mathrm{E} b_{n}^{-1}-1-\varepsilon_{n}\right) r_{n}+\tilde{m}_{*}=O\left(r_{n}^{-1}\right) .
$$

Утверждение 4.4. При $n \rightarrow \infty$

$$
r_{n} \mathbf{E}\left(\frac{b}{b_{n}}-1-\varepsilon_{n}\right)^{2}=1+2\left(\delta_{n}-\varepsilon_{n}\right)-\varepsilon_{n}^{2}-\widetilde{m}^{\prime}+O\left(r_{n}^{-1}\right) .
$$

Отметим, что $\widetilde{m}_{*}=\widetilde{m}^{\prime}=0, \varepsilon_{n}=v_{1}\left(N_{n}\right), \delta_{n}=v_{2}\left(N_{n}\right)$, если положить

$$
N_{i, n}=N_{n} \quad(i \in \mathbf{N}) .
$$

Утверждение 4.5. Если выполнено (4.12), то при $n \rightarrow \infty, N \equiv$ $N_{n} \rightarrow \infty$

$$
\begin{aligned}
(n p(N))^{2} \mathbf{E}\left(\frac{b}{b_{n}}-1-\varepsilon_{n}\right)^{2}= & (n p(N)+1-p(N))\left(1+2\left(\delta_{n}-\varepsilon_{n}\right)-\varepsilon_{n}^{2}\right) \\
& +O\left(\frac{1}{n p(N)}\right) .
\end{aligned}
$$

Из утверждений $4.3,4.5$ следует, что в условиях утверждения 4.5

$$
\begin{aligned}
n p(N) \mathrm{E}\left(\frac{b}{b_{n}}-1\right)^{2}= & 1+2\left(\delta_{n}-\varepsilon_{n}\right)-\varepsilon_{n}^{2}+\varepsilon_{n}^{2} n p(N) \\
& +\frac{1}{n p(N)}+o\left(\frac{1}{n p(N)}\right) .
\end{aligned}
$$

Аналогичные утверждениям 4.1-4.5 результаты могут быть выведены и для статистики, предложенной в [24].

Утверждения 4.1-4.5 следуют из теорем 2.1-2.5.

3 а м е ч а н и е о б о д н о й б лा и 3 к о й 3 а да ч $е$ Пусть $Y_{1}, \ldots, Y_{n}$ - независимые наблюдения над распределением с.в. $Y$, удовлетворяющим условию Крамера: $\mathrm{E} \exp (t Y)<\infty$ для некоторого $t>0$. Положим $\sigma=\sup \{t: \mathbf{E} \exp (t Y)<\infty\}$. В работе [37] рассматривалась задача оценивания числа $\sigma$ по выборке $Y_{1}, \ldots, Y_{n}$ с помощью статистик

$$
\sigma_{n}=\frac{\sum_{i=1}^{n} 1\left\{Y_{i}>y_{n}\right\}}{\sum_{i=1}^{n}\left(Y_{i}-y_{n}\right) 1\left\{Y_{i}>y_{n}\right\}}, \quad \widehat{\sigma}_{n}=\frac{Y_{(n)}}{\ln n},
$$

где $Y_{(n)}=\max \left\{Y_{i}: 1 \leqslant i \leqslant n\right\}$, числа $\left\{y_{n}\right\}$ выбираются исследователем.

В [37] установлено, что при определенных предположениях на распределение с.в. $Y$ оценки $\sigma_{n}, \widehat{\sigma}_{n}$ состоятельны и скорость сходимости $\sigma_{n}$ к $\sigma$ есть $1 / \ln n$.

3 Теория вероятностей и ее применения, № 3 
Покажем, что задача опенивания числа $\sigma$ в известном смысле сводится к задаче оценивания индекса $b$. А именно, определим функцию $L$ равенством

$$
\mathbf{P}\{Y \geqslant y\}=e^{-\sigma y} L\left(e^{y}\right) .
$$

Если $L$ - мецленно меняюшаяся функция, то распределение с.в. $X=e^{Y}$ удовлетворяет (4.1) с $b=\sigma$; условное распределение $Y-y$ при условии $Y \geqslant y$ сходится при $y \rightarrow \infty$ к экспоненциальному закону $\mathbf{E}(\sigma)$; для любого $t \in \mathbf{R}$

$$
h(y)=o(y), \quad h(y+t)-h(y)=o(1) \quad(y \rightarrow \infty),
$$

где $h(y)=\ln L\left(e^{y}\right)$ (в этом нетрудно убедиться с помошью свойств медленно меняюшихся функций).

Таким образом, если предположить, что распределение с.в. $Y$ удовлетворяет (4.14), где $L$ - медленно меняюшаяся функция, то из результатов настояшего раздела вытекают соответствуюшие утверждения об асимптотике распределения оценки $\sigma_{n}$.

5. Статистическое оценивание $f(x) /(1-F(x))$. Пусть дана выборка $X_{1}, \ldots, X_{n}$ независимых наблюдений над распределением с.в. $X$ с функцией распределения $F$ и плотностью $f$. В дальнейшем в этом разделе символ $f_{\xi}$ для любой с.в. $\xi$ обозначает плотность ее распределения, $\bar{F}(x)=1-F(x)$.

Пусть $\bar{F}(x)>0$. Определим функцию интенсивности отказов (иначе - «failure rate» или «hazard function») равенством

$$
h(x)=\frac{f(x)}{\bar{F}(x)} .
$$

Потребность в сведениях о функции $h$ возникает в теории надежности, сейсмологии и других областях (см. [40], [41]). Требуется ощенить число $h(x)$.

Ватсон и Лидбеттер [42] предложили ощенки

$$
\begin{aligned}
h_{n}^{*}(x) & =\frac{f_{n}(x)}{\bar{F}_{n}(x)}, \\
h_{n}^{(2)}(x) & =\sum_{1}^{n} \frac{f_{x+\varepsilon \gamma}\left(X_{i}\right)}{n \bar{F}_{n}\left(X_{i}\right)},
\end{aligned}
$$

где $F_{n}-$ выборочная функция распределения, $\bar{F}_{n}=1-F_{n}, f_{n} \equiv f_{n, \varepsilon}$ есть классическая ядерная оценка плотности с ядром $f_{\gamma}$ (см. также [41], [43]):

$$
f_{n}(x)=\frac{1}{n} \sum_{1}^{n} f_{x+\varepsilon \gamma}\left(X_{i}\right) .
$$


В настояшем разделе предлагается обобщение статистики (5.2) для схемы рекуррентного оценивания.

Положим

$$
h_{n}(x)=\frac{\sum_{1}^{n} f_{x+\varepsilon_{i} \gamma_{i}}\left(X_{i}\right)}{\sum_{1}^{n} 1\left\{X_{i} \geqslant x\right\}}
$$

где $0<\varepsilon_{i} \rightarrow 0$ при $i \rightarrow \infty$, распределения случайных величин $\left\{\gamma_{i}\right\}$ и числа $\left\{\varepsilon_{i}\right\}$ выбираются статистиком. Мы предполагаем, что распределение каждой с.в. $\gamma_{i}$ симметрично и $\mathbf{E} \gamma_{i}^{2}=1$ (иначе от $\varepsilon_{i}$ и $\gamma_{i}$ перейдем к $\varepsilon_{i}^{*}=\varepsilon_{i} \sqrt{\mathbf{D} \gamma_{i}}$ и $\left.\gamma_{i}^{*}=\gamma_{i} / \sqrt{\mathbf{D} \gamma_{i}}\right)$.

Положим

$$
\begin{aligned}
\nu_{i j} & =\mathbf{E} f_{\gamma_{i}}^{j}\left(\gamma_{i}\right), \quad \nu_{i}=\nu_{i 1}, \\
H_{n k t}^{*} & =\sum_{1}^{n} \varepsilon_{i}^{t-k} \mathbf{E} f_{\gamma_{i}}^{k}\left(\gamma_{i}\right) \gamma_{i}^{t}, \quad H_{n k}^{*}=H_{n k 0}^{*}, \\
r_{n i}^{+} & =\left(h(x)+r_{n x}^{(2)}\right)\left(\left\|f^{\prime \prime}\right\| \frac{H_{n 02}^{*}}{2 n}+2\left\|f^{\prime}\right\| \varepsilon_{i} \mathbf{E} \gamma_{i}^{+}\right), \\
q_{n} & =\left\|f^{\prime \prime}\right\| \frac{H_{n 12}^{*}}{2}+\sum_{1}^{n} r_{n i}^{+}, \\
r_{n x}^{(1)} & =\frac{q_{n}+\left\|f^{\prime \prime}\right\|^{2} \sum \varepsilon_{i}^{4}}{f(x) H_{n 1}^{*}}, \quad r_{n x}^{(2)}=\left\|f^{\prime \prime}\right\| \frac{H_{n 02}^{*}}{2 n \mathrm{P}\{X \geqslant x\}},
\end{aligned}
$$

и пусть $G(x)=\left\{n \in \mathbf{N}: r_{n x}^{(1)}<1\right\}$,

$$
\begin{aligned}
q_{n x}^{*} & =\frac{3\|f\|\left\|f^{\prime \prime}\right\|}{\mathbf{P}\{X \geqslant x\}}\left(4\|f\| H_{n 02}^{*}+\sum_{1}^{n} \varepsilon_{i} \nu_{i}+\frac{H_{n 02}^{*} H_{n 1}^{*}}{n}\right), \\
\widehat{r}_{n x} & =\frac{f(x) H_{n 2}^{*}+\left\|f^{\prime \prime}\right\| H_{n 22}^{*} / 2+q_{n x}^{*}}{\left(f(x) H_{n 1}^{*}\right)^{3 / 2}\left(1-r^{(1)}\right)^{3 / 2}}, \\
\check{r}_{n x} & =3 \widehat{r}_{n x}+4(n \mathbf{P}\{X \geqslant x\} \mathbf{P}\{X<x\})^{-1 / 2}, \\
\beta(n, x) & =\frac{\left(f(x) H_{n 1}^{*}\right)^{1 / 2}}{n \mathbf{P}\{X \geqslant x\}}, \quad Q_{n x}^{(i)}=\frac{r_{n x}^{(i)}}{\left(1-r_{n x}^{(i)}\right) \beta(n, x)} \quad(i=2,3), \\
Q_{n x}^{(1)} & =27 C_{*} \frac{\check{r}_{n x}}{8}+8 e^{-1}\left(\frac{\mathbf{P}\{X<x\}}{2 \pi n \mathbf{P}\{X \geqslant x\}}\right)^{1 / 2}+17 \frac{\mathbf{P}\{X<x\}}{n \mathbf{P}\{X \geqslant x\}} .
\end{aligned}
$$

Теорема 5.1. Для пюбого $n \in G(x)$

$$
\begin{gathered}
\sup _{y}\left|\mathbf{P}\left\{h_{n}(x)-h(x)<y \beta(n, x)\right\}-\Phi(y)\right| \\
\leqslant Q_{n x}^{(1)}+Q_{n x}^{(2)}+\frac{r_{n x}^{(1)}}{\left(1-r_{n x}^{(1)}\right) \sqrt{2 \pi e}} .
\end{gathered}
$$


Ниже запись $a_{n}(x) \lesssim b_{n}(x)$ означает, что

$$
a_{n}(x) \leqslant b_{n}(x)(1+o(1)) \quad(n \rightarrow \infty) .
$$

Следствие 5.1. Если $\varepsilon_{i}=\varepsilon u \gamma_{i}=\gamma$ длg любого $i, \varepsilon \equiv \varepsilon(n) \longrightarrow 0$ npu $n \rightarrow \infty$, mo

$$
\begin{aligned}
\Delta_{n}(x) & \equiv \sup _{y}\left|\mathbf{P}\left\{h_{n}^{*}(x)-h(x)<\frac{y \beta_{x}}{\sqrt{2 \pi n \varepsilon}}\right\}-\Phi(y)\right| \\
& \lesssim \frac{81 C_{*} \nu_{*}}{8 \nu^{3 / 2} \sqrt{f(x) n \varepsilon}}+\frac{\left\|f^{\prime \prime}\right\| \sqrt{n \varepsilon^{5}}}{2 \sqrt{2 \pi \nu f(x)}},
\end{aligned}
$$

2de $\nu=\mathbf{E} f_{\gamma}(\gamma), \nu_{*}=\mathbf{E} f_{\gamma}^{2}(\gamma), \beta_{x}=\sqrt{2 \pi \nu f(x)} / \mathbf{P}\{X \geqslant x\}$.

Минимум по $\varepsilon$ в правой части (5.9) достигается при выборе

$$
\varepsilon(n)=\left(\frac{81 C_{*} \nu_{*} \sqrt{2 \pi}}{20 \nu \| f^{\prime \prime}|| n}\right)^{1 / 3}
$$

ІІри этом правая часть (5.9) есть $O_{x}\left(n^{-1 / 3}\right)$. Таков же порядок убывания правой части (5.7) и в схеме рекуррентного ощенивания, если $\varepsilon_{i} \sim c i^{-1 / 3}$, $\gamma_{i}=\gamma$.

Предположим теперь, что $f$ четырежды дифференцируема, и пусть

$$
g_{n}(x)=f^{\prime \prime}(x) \frac{H_{n 02}^{*}}{2 n \mathbf{P}\{X \geqslant x\}}, \quad r_{n x}^{(3)}=\left\|f^{(4)}\right\| \frac{H_{n 04}^{*}}{24 n \mathbf{P}\{X \geqslant x\}} .
$$

Теорема 5.2. Для любого $n \in G(x)$

$$
\begin{aligned}
& \sup _{y}\left|\mathbf{P}\left\{h_{n}(x)-h(x)<y \beta(n, x)\right\}-\Phi\left(y-\frac{g_{n}(x)}{\beta(n, x)}\right)\right| \\
& \quad \leqslant Q_{n x}^{(1)}+Q_{n x}^{(3)}+\frac{r_{n x}^{(1)}}{\left(1-r_{n x}^{(1)}\right) \sqrt{2 \pi e}} .
\end{aligned}
$$

Следствие 5.2. Если $\varepsilon_{i}=\varepsilon$ u $\gamma_{i}=\dot{\gamma} \partial л я$ любого $i, \varepsilon \equiv \varepsilon(n) \longrightarrow 0$ npu $n \rightarrow \infty$, mo

$$
\begin{aligned}
& \sup _{y}\left|\mathbf{P}\left\{h_{n}^{*}(x)-h(x)<\frac{y \beta_{x}}{\sqrt{2 \pi n \varepsilon}}\right\}-\Phi\left(y-f^{\prime \prime}(x) \sqrt{\frac{n \varepsilon^{5}}{4 \nu f(x)}}\right)\right| \\
& \quad \lesssim \frac{\delta_{1}}{\sqrt{n \varepsilon}}+\delta_{2} \varepsilon^{2}+\delta_{3} \sqrt{n \varepsilon^{9}}
\end{aligned}
$$

где функции $\delta_{i} \equiv \delta_{i}(x)$ определены формулами

$$
\begin{aligned}
\delta_{1} & =\frac{81 C_{*} \nu_{*}}{8 \nu^{3 / 2} \sqrt{f(x)}}, \quad \delta_{3}=\left\|f^{(4)}\right\| \frac{\mathbf{E} \gamma^{4}}{24 \sqrt{2 \pi \nu f(x)}} \\
\delta_{2} & =\frac{\left\|f^{\prime \prime}\right\| \mathbf{E} \gamma^{2} f_{\gamma}(\gamma) / 2+2 h(x)\left\|f^{\prime}\right\| \mathbf{E} \gamma^{+}}{\nu f(x) \sqrt{2 \pi e}}
\end{aligned}
$$


Минимум по $\varepsilon$ в правой части $\left(5.9^{*}\right)$ достигается выбором

$$
\varepsilon^{5 / 2}=\frac{2 \delta_{2}}{9 \delta_{3}} \frac{\sqrt{1+9 \delta_{1} \delta_{3} /\left(4 \delta_{2}^{2}\right)}-1}{\sqrt{n}}
$$

При этом точность аппроксимации в $\left(5.9^{*}\right)$ есть $O\left(n^{-2 / 5}\right)$.

Пусть $\gamma_{i} \equiv \gamma$. С помощью теорем $2.3,2.4$ нетрудно показать, что при определенных предположениях на $f$ и $f_{\gamma}$

$$
\mathbf{E} h_{n}(x)-\frac{m_{1}}{m_{2}}=O_{x}\left(\frac{1}{n}\right), \quad \mathbf{E}\left(h_{n}(x)-\frac{m_{1}}{m_{2}}\right)^{2}-\left(\frac{\sigma_{1}}{m_{2}}\right)^{2}=O_{x}\left(n^{-2}\right)
$$

при $n \rightarrow \infty$ (обозначим п. 1) и, следовательно,

$$
\begin{aligned}
\mathbf{E}\left(h_{n}(x)-h(x)\right)^{2}= & \frac{f(x) H_{n 1}^{*}}{(n \mathbf{P}\{X \geqslant x\})^{2}}+\left(\frac{f^{\prime \prime}(x) H_{n 02}^{*}}{2 n \mathbf{P}\{X \geqslant x\}} \mathbf{E} \gamma^{2}\right)^{2} \\
& +O_{x}\left(\left(H_{n 02}^{*}+r_{n x}^{(1)} H_{n 1}^{*}\right) n^{-2}\right)
\end{aligned}
$$

Если $\varepsilon_{i} \equiv \varepsilon, \varepsilon=\varepsilon(n) \longrightarrow 0$ при $n \rightarrow \infty$, то правая часть этого соотношения асимптотически эквивалентна

$$
\frac{\nu h(x)}{n \varepsilon \mathbf{P}\{X \geqslant x\}}+\left(\frac{\varepsilon^{2} f^{\prime \prime}(x) \mathbf{E} \gamma^{2}}{2 \mathbf{P}\{X \geqslant x\}}\right)^{2}
$$

(cp. с [43]). Минимум по $\varepsilon$ в (5.12) достигается при $\varepsilon=\varepsilon_{*}$, где

$$
\varepsilon_{*}^{5}=\varepsilon_{*}^{5}(n, x)=\frac{\nu f(x)}{n\left(f^{\prime \prime}(x) \mathbf{E} \gamma^{2}\right)^{2}}
$$

При $\varepsilon=\varepsilon_{*}$ выражение (5.12) примет вид

$$
\frac{5}{4} n^{-4 / 5}(\mathbf{P}\{X \geqslant x\})^{-2}(\nu f(x))^{4 / 5}\left(f^{\prime \prime}(x) \mathbf{E} \gamma^{2}\right)^{2 / 5}
$$

Доказательство теорем 5.1 и 5.2 аналогично выводу теорем 3.1, 3.2; поэтому детали опущены.

6. Доказательства теорем 2.1-2.2. Положим

$$
\begin{gathered}
m_{*} \equiv m_{*}(n)=\sum \mathbf{E} \xi_{i}^{*} \bar{\eta}_{i}, \quad \xi_{i}(n, x)=\bar{\xi}_{i}^{*}-x d_{n} \frac{\bar{\eta}_{i}}{m_{2}} \\
\mu_{n}(x)=\sum \mathbf{E}\left|\xi_{i}(n, x)\right|^{3} \\
d_{n} \equiv d_{n}(x)=\sigma_{1}\left(\frac{x m_{*}}{\sigma_{1} m_{2}}+\sqrt{1-\left(\frac{x \sigma_{2}}{m_{2}}\right)^{2}+\left(\frac{x m_{*}}{\sigma_{1} m_{2}}\right)^{2}}\right)^{-1} \\
B_{n}^{*}=\left\{x \in \mathbf{R}:\left|x \sigma_{2}\right| \leqslant\left|\frac{m_{2}}{2}\right|\right\}
\end{gathered}
$$


Отметим, что число $d_{n}(x)$ корректно определено при всех $x$ таких, что $\left|x \sigma_{2} / m_{2}\right| \leqslant 1$.

В доказательстве теорем 2.1, 2.2 используются известные равномерная и неравномерная оценки скорости сходимости в ЦПТ. Основная идея состоит в введении случайных величин $\xi_{i}(n, x)$, к распределениям которых и применяются упомянутые оценки. Причем число $d_{n}$ выбирается так, что $d_{n}^{2}=\mathrm{D} \sum \xi_{i}(n, x)$. Второй этап доказательства посвящен учету погрешностей, связанных с приближением $d_{n} \approx \sigma_{1}$.

Лемма 6.1. При $n \geqslant 1, x \in B_{n}^{*}$

$$
\begin{aligned}
\Delta^{*}(n, x) & \equiv\left|\mathbf{P}\left\{\sum\left(\xi_{i}-\frac{\eta_{i} m_{1}}{m_{2}}\right)<x d_{n} \frac{\sum \eta_{i}}{m_{2}}\right\}-\Phi(x)\right| . \\
& \leqslant C_{+}\left(1+|x|^{3}\right)^{-1} d_{n}^{-3} \mu,
\end{aligned}
$$

где $C_{+}=31.995$ есть абсолютная константа.

Д ок а з а т е л с с в о. Заметим, что

$$
\left\{\sum\left(\xi_{i}-\frac{\eta_{i} m_{1}}{m_{2}}\right)<x d_{n} \sum \frac{\eta_{i}}{m_{2}}\right\}=\left\{\sum\left(\xi_{i}^{*}-x d_{n} \frac{\bar{\eta}_{i}}{m_{2}}\right)<x d_{n}\right\} .
$$

Число $d_{n}$ выбрано нами как решение квадратного (относительно $d_{n}$ ) уравнения

$$
d_{n}^{2}=\sum \mathrm{D}\left(\bar{\xi}_{i}^{*}-x d_{n} \frac{\bar{\eta}_{i}}{m_{2}}\right)
$$

Следующий результат доказан в [11].

Теорема С. Предположим, что случайные величины $X_{1}, \ldots, X_{n}$ независимы и имеют нулевое среднее; $G_{n}=X_{1}+\cdots+X_{n}$. Тогда для любого $n \geqslant 1, x \in \mathbf{R}$

$$
\left(1+|x|^{3}\right)\left|\mathbf{P}\left\{G_{n}<x \sqrt{\mathbf{D} G_{n}}\right\}-\Phi(x)\right| \leqslant C_{+} \sum \mathbf{E}\left|X_{i}\right|^{3}\left(\mathbf{D} G_{n}\right)^{-3 / 2} .
$$

Из (6.4)-(6.6) выводим:

$$
\Delta^{*}(n, x) \leqslant C_{+} d_{n}^{-3} \mu_{n}(x)\left(1+|x|^{3}\right)^{-1} .
$$

С помощью формулы Тейлора заключаем, что

$$
\mathbf{E}\left|\xi_{i}(n, x)\right|^{3} \leqslant \mathbf{E}\left|\bar{\xi}_{i}^{*}\right|^{3}+3\left|x d_{n}\right| \mathbf{E}\left|\frac{\bar{\eta}_{i}}{m_{2}}\right|\left(\bar{\xi}_{i}^{*}\right)^{2}+3 \mathbf{E}\left|x d_{n} \frac{\bar{\eta}_{i}}{m_{2}}\right|^{3} .
$$

Из (6.2) и приводимой ниже леммы 6.2 (см. $\left.\left(6.10^{\prime}\right)\right)$ следует, что

$$
\left|x d_{n}\right| \leqslant \frac{\sigma_{1}\left|m_{2}\right|}{\sigma_{2}} \quad\left(x \in B_{n}^{*}\right) .
$$

Применяя неравенство Гёльдера, из (6.7)-(6.9) получим (6.3). 
Лемма 6.1 доказана.

Лемма 6.2. Для любого $x \in B_{n}^{*}$

$$
\left|1-\frac{\sigma_{1}}{d_{n}}\right| \leqslant\left|\frac{x \sigma_{2}}{m_{2}}\right| \leqslant \frac{1}{2} .
$$

Д о к а з а т е л с с т о. Равенство (6.1) можно переписать в виде

$$
\frac{\sigma_{1}}{d_{n}}=\frac{x m_{*}}{\sigma_{1} m_{2}}+\sqrt{1-\left(\frac{x \sigma_{2}}{m_{2}}\right)^{2}+\left(\frac{x m_{*}}{\sigma_{1} m_{2}}\right)^{2}} .
$$

Соотношение $(6.11)$ и очевидное неравенство

$$
\left|m_{*}\right| \leqslant \sigma_{1} \sigma_{2}
$$

влекут за собой неравенство

$$
\frac{\sigma_{1}}{d_{n}} \leqslant 1+\frac{\left|x m_{*}\right|}{\sigma_{1} m_{2}} \leqslant 1+\left|\frac{x \sigma_{2}}{m_{2}}\right|
$$

при всех $x$ таких, что $\left|x \sigma_{2} / m_{2}\right| \leqslant 1$. Отметим, что для любого $\varepsilon \geqslant$ $\left|x \sigma_{2} / m_{2}\right|$ неравенство $\sigma_{1} / d_{n} \geqslant \varepsilon$ эквивалентно следующему:

$$
1-\left(\frac{x \sigma_{2}}{m_{2}}\right)^{2}\left\{1-\left(\frac{m_{*}}{\sigma_{1} \sigma_{2}}\right)^{2}\right\} \geqslant\left(\varepsilon-\frac{x m_{*}}{\sigma_{1} m_{2}}\right)^{2},
$$

что может быть переписано в виде

$$
1-\left(\frac{x \sigma_{2}}{m_{2}}\right)^{2} \geqslant \varepsilon^{2}-2 \varepsilon \frac{x m_{*}}{\sigma_{1} m_{2}}
$$

Но правая часть (6.14) не превосходит $\varepsilon^{2}+2 \varepsilon\left|x \sigma_{2} / m_{2}\right|$. Полагая $\varepsilon=$ $1-\left|x \sigma_{2} / m_{2}\right|$, получим:

$$
\frac{\sigma_{1}}{d_{n}} \geqslant 1-\left|\frac{x \sigma_{2}}{m_{2}}\right|
$$

Из (6.2), (6.13), (6.15) вытекает (6.10).

Лемма 6.2 доказана.

Следствие 6.1. Iр $u \geqslant 1, x \in B_{n}^{*}$

$$
\left|\Phi\left(\frac{x \sigma_{1}}{d_{n}}\right)-\Phi(x)\right| \leqslant x^{2} \varphi\left(\frac{x}{2}\right) \frac{\sigma_{2}}{\left|m_{2}\right|} .
$$

Д о к а з а т е л ь с т в о. Согласно (6.10), имеет место неравенство

$$
\frac{1}{2} \leqslant \frac{\sigma_{1}}{d_{n}} \leqslant \frac{3}{2}
$$


С помощью $\left(6.10^{\prime}\right)$ и формулы Тейлора выводим:

$$
\begin{aligned}
& \left|\Phi\left(\frac{x \sigma_{1}}{d_{n}}\right)-\Phi(x)\right| \leqslant|x|\left|1-\frac{\sigma_{1}}{d_{n}}\right| \varphi\left(|x| \min _{0 \leqslant \theta \leqslant 1}\left\{\theta+(1-\theta) \frac{\sigma_{1}}{d_{n}}\right\}\right) \\
& \quad \leqslant|x|\left|1-\frac{\sigma_{1}}{d_{n}}\right| \varphi\left(\frac{x}{2}\right),
\end{aligned}
$$

что вместе с (6.10) влечет за собой (6.16).

Следствие 6.1 доказано.

Доказ а тельст во те оремы 2.2 . Ввиду $\left(6.10^{\prime}\right)$, $x \sigma_{1} / d_{n} \in B_{n}^{*}$ как только $x \in B_{n}$. Из леммы 6.1 и $\left(6.10^{\prime}\right)$ находим, что при $x \in B_{n}$

$$
\left|\mathbf{P}\left\{\sum\left(\xi_{i}-\frac{\eta_{i} m_{1}}{m_{2}}\right)<x \sigma_{1} \frac{\sum \eta_{i}}{m_{2}}\right\}-\Phi\left(\frac{x \sigma_{1}}{d_{n}}\right)\right| \leqslant C_{+}\|x\|_{*}^{-3} \sigma_{1}^{-3} \mu,
$$

что вместе с (6.16) влечет за собой утверждение теоремы 2.2 .

Д о к аз а те ль с т в о т е о ре мы 2.1 проводится аналогично доказательству теоремы 2.2 с использованием вместо теоремы $\mathbf{C}$ результата Жиганова [12], что вместе с (6.10) дает оценку

$$
\begin{aligned}
& \left|\mathbf{P}\left\{\sum\left(\xi_{i}-\frac{\eta_{i} m_{1}}{m_{2}}\right)<x \sigma_{1} \sum \frac{\eta_{i}}{m_{2}}\right\}-\Phi\left(\frac{x \sigma_{1}}{d_{n}}\right)\right| \\
& \quad \leqslant C_{*} \mu \sigma_{1}^{-3}\left(1+\frac{|x| \sigma_{2}}{m_{2}}\right)^{3} \leqslant C_{*} \mu\left(\frac{3}{2 \sigma_{1}}\right)^{3}
\end{aligned}
$$

при $x \in B_{n}$. Заметим, что

$$
\sup _{x} x^{2} \varphi\left(\frac{x}{2}\right)=\frac{8}{e \sqrt{2 \pi}} .
$$

Оценим

$$
\sup _{x \notin B_{n}}\left|\mathbf{P}\left\{\sum \xi_{i}^{*}<x \sigma_{1} \sum \frac{\eta_{i}}{m_{2}}\right\}-\Phi(x)\right| .
$$

Для определенности будем считать, что $m_{2}>0$. Ввиду (6.2) и неравенства Чебышева,

$$
\begin{aligned}
& \mathbf{P}\left\{\sum \xi_{i}^{*} \geqslant x \sigma_{1} \sum \frac{\eta_{i}}{m_{2}}\right\} \leqslant \mathbf{P}\left\{\sum \frac{\xi_{i}^{*}}{\sigma_{1}} \geqslant \sum \frac{\eta_{i}}{3 \sigma_{2}} ; \sum \eta_{i} \geqslant 0\right\} \\
& +\mathbf{P}\left\{\sum \eta_{i}<0\right\} \leqslant \mathbf{P}\left\{\sum\left(\frac{\xi_{i}^{*}}{\sigma_{1}}-\frac{\bar{\eta}_{i}}{3 \sigma_{2}}\right) \geqslant \frac{m_{2}}{3 \sigma_{2}}\right\} \\
& +\mathbf{P}\left\{\sum \bar{\eta}_{i}<-m_{2}\right\} \leqslant 17\left(\frac{\sigma_{2}}{m_{2}}\right)^{2}
\end{aligned}
$$


при $x \in \mathbf{R}^{+} \backslash B_{n}$. Для нормальной $\mathcal{N}(0,1)$ с.в. $\zeta$ выполняется:

$$
\mathbf{P}\left\{\zeta \in \mathbf{R}^{+} \backslash B_{n}\right\} \leqslant 9\left(\frac{\sigma_{2}}{m_{2}}\right)^{2} .
$$

Объединяя (6.16), (6.19)-(6.21), получим (2.2).

Теорема 2.1 доказана.

Отметим, что константы в неравенствах (2.2), (2.3) не оптимальны. Более точные ощенки можно получить, если вместо $B_{n}$ взять, к примеру, множество $\left\{x:|x| \leqslant\left(\left|m_{2}\right| / \sigma_{2}\right)^{\alpha}\right\}, \alpha \in(0 ; 1)$.

7. Доказательства теорем 2.3-2.5. Положим

$$
\begin{aligned}
\zeta_{n} & =\frac{\bar{T}_{n}}{m_{2}}, \quad \zeta_{n i}=\frac{\bar{T}_{n i}}{m_{2}}, \quad \bar{T}_{n i}=T_{n}-\eta_{i} \\
\tau_{n} & =1\left\{T_{n}>m^{+}\right\}, \quad \tau_{n i}=1\left\{T_{n i}>m^{+}\right\}, \quad \tau_{n i}^{*}=1-\tau_{n i}
\end{aligned}
$$

аналогично определяются $\tau_{n}^{*}$ и $\tau_{n i j}^{*}$.

Д о к а 3 а т е л ь с т в т е о е м ы 2.3 опирается на следующее соотношение:

$$
m_{2} \mathbf{E} Z_{n}-m_{1}=m_{2} \mathbf{E} \frac{S_{n}^{*}}{T_{n}}=\sum \mathbf{E} \xi_{i}^{*}\left(1+\frac{\bar{\eta}_{i}}{m_{2}}+\zeta_{n i}\right)^{-1}
$$

Пользуясь тождеством

$$
(1+x)^{-1}=1-x+x^{2}(1+x)^{-1}
$$

с $x=\bar{\eta}_{i} /\left(m_{2}\left(1+\zeta_{n i}\right)\right)$, получим:

$$
\begin{aligned}
& \mid \sum \mathbf{E} \xi_{i}^{*}\left(1+\frac{\bar{\eta}_{i}}{m_{2}}+\zeta_{n i}\right)^{-1} \tau_{n i}-\sum \mathbf{E} \xi_{i}^{*} \mathbf{E}\left(1+\zeta_{n i}\right)^{-1} \tau_{n i} \\
& +\sum \mathbf{E} \xi_{i}^{*} \bar{\eta}_{i} \mathbf{E}\left(1+\zeta_{n i}\right)^{-2} \frac{\tau_{n i}}{m_{2}}\left|\leqslant \delta^{-3} m_{2}^{-2} \sum \mathbf{E}\right| \xi_{i}^{*} \mid \eta_{i}^{2} \equiv \rho_{1}
\end{aligned}
$$

Заметим, что

$$
\begin{aligned}
\left|\sum \mathbf{E} \xi_{i}^{*} \mathbf{E}\left(1+\zeta_{n i}\right)^{-1} \tau_{n i}\right| \leqslant & 3 \delta^{-3} m_{2}^{-2} \sum\left|\mathbf{E} \xi_{i}^{*}\right| \mathbf{D} \eta_{i} \\
& +\delta^{-1} \alpha(n) \sum\left|\mathbf{E} \xi_{i}^{*}\right| \equiv \rho_{2}
\end{aligned}
$$

Действительно,

$$
1+\zeta_{n i}=1+\zeta_{n}-\frac{\bar{\eta}_{i}}{m_{2}}=\left(1+\zeta_{n}\right)\left(1-\frac{\bar{\eta}_{i}}{m_{2}\left(1+\zeta_{n}\right)}\right)
$$


С учетом (7.2) выводим:

$$
\begin{aligned}
& \mid \sum \mathbf{E} \xi_{i}^{*} \mathbf{E}\left(1+\zeta_{n i}\right)^{-1} \tau_{n i}-\sum \mathbf{E} \xi_{i}^{*} \mathbf{E}\left(1+\zeta_{n}\right)^{-1} \tau_{n i} \\
& -\sum \mathbf{E} \xi_{i}^{*} \mathbf{E} \frac{\bar{\eta}_{i} \tau_{n i}}{\left(1+\zeta_{n}\right)^{2} m_{2}}\left|\leqslant \delta^{-3} m_{2}^{-2} \sum\right| \mathbf{E} \xi_{i}^{*} \mid \mathbf{D} \eta_{i} .
\end{aligned}
$$

Далее, заметим, что

$$
\tau_{n} \equiv 1\left\{T_{n}>m^{+}\right\}=\tau_{n i}+1\left\{T_{n i} \leqslant m^{+}<T_{n}\right\} .
$$

Отсюда и из приводимой ниже леммы 7.2 вытекает оценка

$$
\left|\mathbf{E}\left(1+\zeta_{n}\right)^{-1} \tau_{n i}-\mathbf{E}\left(1+\zeta_{n}\right)^{-1} \tau_{n}\right| \leqslant \delta^{-1} \alpha(n) .
$$

Учитывая, что

$$
(1+x)^{-2}=1-x(1+x)^{-1}-x(1+x)^{-2} \quad \text { и } \quad 1+\zeta_{n}=1+\zeta_{n i}+\frac{\bar{\eta}_{i}}{m_{2}},
$$

заключаем, что

$$
\tau_{n i}\left|\left(1+\zeta_{n}\right)^{-2}-\left(1+\zeta_{n i}\right)^{-2}\right| \leqslant 2 \delta^{-3}\left|\frac{\bar{\eta}_{i}}{m_{2}}\right|
$$

Из (7.5)-(7.7) и тождества $\sum \mathbf{E} \xi_{i}^{*}=\sum \mathbf{E} \xi_{i}^{*} \mathbf{E}\left(1+\zeta_{n}\right)^{-1} \tau_{n}=0$ выводим (7.4).

Вследствие приводимой ниже леммы 7.2 справедлива оценка

$$
\left|\sum \mathbf{E} \xi_{i}^{*}\left(1+\frac{\bar{\eta}_{i}}{m_{2}}+\zeta_{n i}\right)^{-1} \tau_{n i}^{*}\right| \leqslant m_{2} \alpha(n) \sum \mathbf{E}\left|\frac{\xi_{i}^{*}}{\eta_{(i)}}\right| \equiv \rho_{3} .
$$

Ввиду неравенства $\left|\zeta_{n i}\right| \tau_{n i}^{*} \leqslant \tau_{n i}^{*}$ и леммы 7.2 имеем:

$$
\left|\mathbf{E} \zeta_{n i} \tau_{n i}\right| \leqslant \alpha(n) \text {. }
$$

Заметим, что $(1+x)^{-2}=1-2 x+2 x^{2}(1+x)^{-1}+x^{2}(1+x)^{-2}$. Следовательно,

$$
\left|\mathbf{E}\left(1+\zeta_{n i}\right)^{-2} \tau_{n i}-1\right| \leqslant 3 \alpha(n)+3\left(\frac{\sigma_{2}}{m^{+}}\right)^{2} \equiv \rho^{*}(n) .
$$

Пусть

$$
\rho_{4}=\rho^{*}(n) \frac{\sum\left|\mathbf{E} \xi_{i}^{*} \bar{\eta}_{i}\right|}{m_{2}} .
$$

Из (7.1)-(7.4), (7.8)-(7.10) вытекает утверждение теоремы 2.3.

Док а з а те льст т о те орем ы 2.4. Положим

$$
T_{n i j}=T_{n}-\eta_{i}-\eta_{j} 1\{i \neq j\}, \quad \zeta_{n i j}=\frac{\bar{T}_{n i j}}{m_{2}}, \quad \tau_{n i j}=1\left\{T_{n i j}>m^{+}\right\}
$$


Таким образом, $\tau_{n i j}=\tau_{n i}$ при $i=j$. Доказательство опирается на тӧждество

$$
\begin{aligned}
\left(m_{2} Z_{n}-m_{1}\right)^{2} \equiv & \left(\frac{m_{2} S_{n}^{*}}{T_{n}}\right)^{2}=\sum_{i}\left(\xi_{i}^{*}\right)^{2}\left(1+\frac{\bar{\eta}_{i}}{m_{2}}+\zeta_{n i}\right)^{-2} \\
& +\sum_{i \neq j} \xi_{i}^{*} \xi_{j}^{*}\left(1+\zeta_{n}\right)^{-2}
\end{aligned}
$$

Рассмотрим сумму

$$
\sum \mathbf{E}\left(\bar{\xi}_{i}^{*}\right)^{2}\left(1+\frac{\bar{\eta}_{i}}{m_{2}}+\zeta_{n i}\right)^{-2} \tau_{n i}+\sum_{i \neq j} \mathbf{E} \bar{\xi}_{i}^{*} \bar{\xi}_{j}^{*}\left(1+\zeta_{n}\right)^{-2} \tau_{n i j}
$$

Заметим, что при $i \neq j$

$$
\begin{aligned}
\lambda_{i j} & \equiv \mathbf{E} \bar{\xi}_{i}^{*} \bar{\xi}_{j}^{*}\left(1+\frac{\bar{\eta}_{i}+\bar{\eta}_{j}}{m_{2}}+\zeta_{n i j}\right)^{-2} \tau_{n i j} \\
& =-2 \mathbf{E} \bar{\xi}_{i}^{*} \bar{\xi}_{j}^{*} \bar{\eta}_{j}\left(1+\frac{\bar{\eta}_{i}+\theta_{j} \bar{\eta}_{j}}{m_{2}}+\zeta_{n i j}\right)^{-3} \frac{\tau_{n i j}}{m_{2}} \\
& =6 \mathbf{E} \bar{\xi}_{i}^{*} \bar{\eta}_{i} \bar{\xi}_{j}^{*} \bar{\eta}_{j}\left(1+\frac{\theta_{i} \bar{\eta}_{i}+\theta_{j} \bar{\eta}_{j}}{m_{2}}+\zeta_{n i j}\right)^{-4} \frac{\tau_{n i j}}{m_{2}^{2}}
\end{aligned}
$$

где каждая из случайных величин $\theta_{j}$ независима от всех прочих случайных величин и имеет равномерное распределение на $[0,1]$. Поэтому

$$
\sum_{i \neq j}\left|\lambda_{i j}\right| \leqslant 6 \delta^{-4} m_{2}^{-2}\left(\sum \mathrm{E}\left|\bar{\xi}_{i}^{*} \bar{\eta}_{i}\right|\right)^{2} \equiv R_{1}
$$

Легко видеть, что

$$
\begin{aligned}
& \left|\sum\left\{\mathbf{E}\left(\bar{\xi}_{i}^{*}\right)^{2}\left(1+\frac{\bar{\eta}_{i}}{m_{2}}+\zeta_{n i}\right)^{-2} \tau_{n i}-\left(\mathbf{D} \xi_{i}^{*}\right) \mathbf{E}\left(1+\zeta_{n}\right)^{-2} \tau_{n i}\right\}\right| \\
& \leqslant 2 \delta^{-3} \sum \mathbf{E}\left(\bar{\xi}_{i}^{*}\right)^{2}\left|\frac{\bar{\eta}_{i}}{m_{2}}\right| \equiv R_{2} .
\end{aligned}
$$

Из (7.9) и (7.16) выводим:

$$
\begin{aligned}
\left|\sum\left\{\mathrm{E}\left(\bar{\xi}_{i}^{*}\right)^{2}\left(1+\frac{\bar{\eta}_{i}}{m_{2}}+\zeta_{n i}\right)^{-2} \tau_{n i}-\mathbf{D} \xi_{i}^{*}\right\}\right| & \leqslant R_{2}(n)+\rho^{*}(n) \sigma_{1}^{2} \\
& \equiv R_{2}+R_{3}
\end{aligned}
$$

Ввиду леммы 7.2 ,

$$
\left|\sum \mathbf{E}\left(\xi_{i}^{*}\right)^{2}\left(1+\frac{\bar{\eta}_{i}}{m_{2}}+\zeta_{n i}\right)^{-2} \tau_{n i}^{*}+\sum_{i \neq j} \mathbf{E} \xi_{i}^{*} \xi_{j}^{*}\left(1+\zeta_{n}\right)^{-2} \tau_{n i j}^{*}\right|
$$




$$
\leqslant m_{2}^{2} \alpha(n)\left(\left(\sum_{i} \mathbf{E}\left|\frac{\xi_{i}^{*}}{\eta_{(i)}}\right|\right)^{2}+\sum_{i} \sum_{j}^{*} \mathbf{E}\left|\xi_{i}^{*} \xi_{j}^{*}\right| \eta_{i j}^{-2}\right) \equiv R_{5}
$$

где суммирование под знаком $\sum_{j}^{*}$ ведется по множеству $\{j$ : $\left.A_{j} \cap A_{i} \neq \varnothing\right\}$. Объединяя (7.12) - (7.18) и учитывая приводимую ниже лемму 7.1 , получим (2.6).

Теорема 2.4 доказана.

Доказ а т е ль с т в о т е о е м 2.5 использует тот факт, что $\mathbf{E} \bar{\xi}_{i}^{*}=0 .(i \geqslant 1)$. Вследствие (7.14),

$$
\begin{aligned}
& \sum_{i \neq j}\left|\lambda_{i j}-6 m_{2}^{-2} \mathbf{E} \bar{\xi}_{i}^{*} \bar{\eta}_{i} \mathbf{E} \bar{\xi}_{j}^{*} \bar{\eta}_{j} \mathbf{E}\left(1+\zeta_{n i j}\right)^{-4} \tau_{n i j}\right| \\
& \quad \leqslant 24 m_{2}^{-3} \delta^{-5}\left(\sum_{i} \mathbf{E}\left|\bar{\xi}_{i}^{*} \bar{\eta}_{i}\right|\right)\left(\sum_{j} \mathbf{E}\left|\bar{\xi}_{j}^{*}\left(\bar{\eta}_{j}\right)^{2}\right|\right) \equiv R_{6} .
\end{aligned}
$$

Отметим, что $\mathbf{E} \zeta_{n i j}=0$ и

$$
\begin{gathered}
(1+x)^{-4}-1+4 x=x^{2}\left\{(1+x)^{-4}+2(1+x)^{-3}+3(1+x)^{-2}\right. \\
\left.+4(1+x)^{-1}\right\}
\end{gathered}
$$

при $x>-1$. С учетом (7.20) и леммы 7.2 имеем:

$$
\left|\mathbf{E}\left(1+\zeta_{n i j}\right)^{-4} \tau_{n i j}-1\right| \leqslant 5 \alpha(n)+10 \delta^{-4}\left(\frac{\sigma_{2}}{m_{2}}\right)^{2} \equiv R_{7}^{*}
$$

где $R_{7}^{*} \equiv R_{7}^{*}(n)$. Следовательно,

$$
\begin{aligned}
& \left|\sum_{i \neq j} \lambda_{i j}-6 m_{2}^{-2}\left(\sum \mathbf{E} \xi_{i}^{*} \bar{\eta}_{i}\right)^{2}\right| \leqslant R_{6}+6 m_{2}^{-2}\left(\sum_{i}\left|\mathbf{E} \bar{\xi}_{i}^{*} \eta_{i}\right|\right)^{2} R_{7}^{*} \\
& \quad+6 m_{2}^{-2} \sum_{i}\left(\mathbf{E} \bar{\xi}_{i}^{*} \eta_{i}\right)^{2} \equiv R_{6}+R_{7}
\end{aligned}
$$

Рассмотрим первое слагаемое в правой части (7.13). Нетрудно проверить, что

$$
\begin{aligned}
& \sum\left(\mathbf{E}\left(\bar{\xi}_{i}^{*}\right)^{2}\left(1+\frac{\bar{\eta}_{i}}{m_{2}}+\zeta_{n i}\right)^{-2} \tau_{n i}-\left(\mathbf{D} \xi_{i}^{*}\right) \mathbf{E}\left(1+\zeta_{n i}\right)^{-2} \tau_{n i}\right. \\
& \left.\quad+2 \mathbf{E}\left(\bar{\xi}_{i}^{*}\right)^{2}\left(\frac{\bar{\eta}_{i}}{m_{2}}\right) \mathbf{E}\left(1+\zeta_{n i}\right)^{-3} \tau_{n i}\right) \\
& =3 \sum \mathbf{E}\left(\frac{\bar{\xi}_{i}^{*} \bar{\eta}_{i}}{m_{2}}\right)^{2}\left(1+\frac{\theta_{i}^{\prime} \bar{\eta}_{i}}{m_{2}}+\zeta_{n i}\right)^{-4} \tau_{n i} \\
& \leqslant 3 \delta^{-4} \sum \mathbf{E}\left(\frac{\bar{\xi}_{i}^{*} \bar{\eta}_{i}}{m_{2}}\right)^{2} \equiv R_{8}
\end{aligned}
$$


H

$$
\left|\mathbf{E}\left(1+\zeta_{n i}\right)^{-3} \tau_{n i}-1\right| \leqslant 4 \alpha(n)+6 \delta^{-3}\left(\frac{\sigma_{2}}{m_{2}}\right)^{2} \equiv \rho_{1}^{*}
$$

(cp. с $\left.\left(7.9^{*}\right)\right)$. Аналогично убеждаемся, что

$$
\begin{aligned}
\mid \mathbf{E}(1 & \left.+\zeta_{n i}\right)^{-2} \tau_{n i}-\left\{1+3\left(\frac{\sigma_{2}}{m_{2}}\right)^{2}\right\} \mid \leqslant 10 \alpha(n) \\
& +3 \mathbf{E}\left(\frac{\bar{\eta}_{i}}{m_{2}}\right)^{2}+4 m_{2}^{-3} \sum\left|\mathbf{E}\left(\bar{\eta}_{i}\right)^{3}\right| \\
& +5 \delta^{-6} m_{2}^{-4}\left\{3 \sigma_{2}^{4}+\sum \mathbf{E}\left(\bar{\eta}_{j}\right)^{4}\right\} \equiv R_{9}(n, i) .
\end{aligned}
$$

Положим

$$
R_{9}=\sum_{i} R_{9}(n, i) \mathbf{D} \xi_{i}^{*}, \quad R_{10}=2 \sum_{i} \mathbf{E}\left|\left(\bar{\xi}_{i}^{*}\right)^{2} \frac{\bar{\eta}_{i}}{m_{2}}\right| \rho_{1}^{*}
$$

Tогда

$$
\begin{aligned}
\sum_{i} \mid \mathbf{E}\left(\bar{\xi}_{i}^{*}\right)^{2}\left(1+\frac{\bar{\eta}_{i}}{m_{2}}+\zeta_{n i}\right)^{-2} \tau_{n i}-\left(\mathbf{D} \xi_{i}^{*}\right)\left\{1+3\left(\frac{\sigma_{2}}{m_{2}}\right)^{2}\right\} \\
\quad+2 \mathbf{E}\left(\bar{\xi}_{i}^{*}\right)^{2} \frac{\bar{\eta}_{i}}{m_{2}} \mid \leqslant R_{8}+R_{9}+R_{10} .
\end{aligned}
$$

Объединяя $(7.13),(7.18),(7.21)-(7.26)$ и учитывая $(7.12)$ и приводимую ниже лемму 7.1 , получим $\left(2.6^{*}\right)$.

Теорема 2.5 доказана.

Лемма 7.1. Справедлива оченка

$$
\begin{aligned}
& \left|\sum_{i, j} \mathbf{E}\left\{\xi_{i}^{*} \xi_{j}^{*}-\bar{\xi}_{i}^{*} \bar{\xi}_{j}^{*}\right\}\left(1+\zeta_{n}\right)^{-2} \tau_{n i j}\right| \\
& \quad \leqslant \delta^{-2} \alpha(n) \sum\left|\mathbf{E} \xi_{j}^{*}\right| \sum \mathbf{E}\left|2 \xi_{i}^{*}-\mathbf{E} \xi_{i}^{*}\right| \equiv R_{4}(n) .
\end{aligned}
$$

Доказательст во ле м м ы 7.1. Воспользуемся тем фактом, что

$$
\sum \xi_{i}^{*}=\sum \bar{\xi}_{i}^{*}
$$

Имеем:

$$
\begin{aligned}
\sum_{i, j} \xi_{i}^{*} \xi_{j}^{*} \tau_{n i j} & =\sum_{i, j} \xi_{i}^{*} \xi_{j}^{*}\left(\tau_{n i j}-\tau_{n}\right)+\sum_{i, j} \bar{\xi}_{i}^{*} \bar{\xi}_{j}^{*} \tau_{n} \\
& =\sum_{i, j} \bar{\xi}_{i}^{*} \bar{\xi}_{j}^{*} \tau_{n i j}+\sum_{i, j}\left\{\xi_{i}^{*} \xi_{j}^{*}-\bar{\xi}_{i}^{*} \bar{\xi}_{j}^{*}\right\}\left(\tau_{n i j}-\tau_{n}\right) \\
& =\sum_{i, j} \bar{\xi}_{i}^{*} \bar{\xi}_{j}^{*} \tau_{n i j}+\sum_{i, j}\left(\tau_{n i j}-\tau_{n}\right)\left\{2 \xi_{i}^{*}-\mathbf{E} \xi_{i}^{*}\right\} \mathbf{E} \xi_{j}^{*}
\end{aligned}
$$


Из (7.28), (7.30) и равенства

$$
\tau_{n i j}=\tau_{n}-1\left\{T_{n i j} \leqslant m^{+}<T_{n}\right\}
$$

следует (7.27).

Лемма 7.1 доказана.

Положим

$$
\begin{aligned}
K \equiv K(n) & =\sum \frac{\mathbf{E} \eta_{i}^{2}}{m^{+}}, \quad \eta_{i}^{*}=\eta_{i} 1\left\{\eta_{i} \leqslant K\right\}, \\
\widehat{T}_{n i j} & =\sum_{k \notin A(i, j)} \eta_{k}, \quad T_{n i j}^{*}=\sum_{k \notin A(i, j)} \eta_{k}^{*} .
\end{aligned}
$$

Лемма 7.2. Для пюбых $i, j \in J_{n}$

$$
\mathbf{P}\left\{\widehat{T}_{n i j} \leqslant m^{+}\right\} \leqslant \exp \left\{-\frac{\left(m^{+}\right)^{2}}{8 \sum \mathbf{E} \eta_{i}^{2}}\right\} \equiv \alpha(n) .
$$

Доказ а тельст во ле м мы 7.2. Заметим, что

$$
\min _{i, j \in J_{n}} \mathbf{E} T_{n i j}=3 m^{+}, \min _{i, j \in J_{n}} \mathbf{E} T_{n i j}^{*} \geqslant 2 m^{+} .
$$

ІІоследнее неравенство справедливо ввиду следуюшей оценки:

$$
\sum \mathbf{E} \eta_{i} 1\left\{\eta_{i}>K\right\} \leqslant \sum \frac{\mathbf{E} \eta_{i}^{2}}{K}=m^{+} .
$$

Поэтому

$$
\left\{\widehat{T}_{n i j} \leqslant m^{+}\right\} \subset\left\{T_{n i j}^{*} \leqslant m^{+}\right\} \subset\left\{T_{n i j}^{*}-\mathbf{E} T_{n i j}^{*} \leqslant-m^{+}\right\} .
$$

Легко видеть, что

$$
\mathbf{D} T_{n i j}^{*} \leqslant \sum \mathbf{E}\left(\eta_{i}^{*}\right)^{2} \leqslant K m^{+} .
$$

Применяя неравенство Бернштейна (см. [38, гл. 3, теорема 18]) к вероятностям событий в правой части (7.31), получим (7.30).

Лемма 7.2 доказана.

Доказательства следствий раздела 2 опускаются ввиду простоты. Достигнутое в них ослабление моментных условий по сравнению с соответствующими результатами [4] объясняется тем, что в настоящей работе мы раскладываем $\left(1+\zeta_{n}\right)^{-\tau}$ в ряд Тейлора по стеленям $\bar{\eta}_{i} / m_{2}$, а не по степеням $\zeta_{n i}$.

Автор благодарен рецензенту за многочисленные замечания, позволившие улучшить изложение и устранить ряд неточностей, присутствовавших в первоначальном варианте статьи. 


\section{СПИСОК ЛИТЕРАТУРЫ}

1. Uspensky J. V. Problem № 3408. - Amer. Math. Monthly, 1932, v. 39, № 1, p. 176177.

2. Kimboll B. F. Problem № 3460. - Amer. Math. Monthly, 1930, v. 37, p. 508.

3. Stoyanov $Y$. Probabilistic proof of the convergence of a class of $n$-fold integrals. Glasnic Math., Ser. III, 1986, v. 21, № 1, p. 101-114.

4. Новак С. Ю., Утев С. А. Об асимптотике распределения отношения сумм случайных величин. - Сиб. матем. ж., 1990, т. 31, № 5, с. 92-101.

5. Надарая Э. А. Об оценке регрессии. - Теория вероятн. и ее примен., 1964, т. IX, B. 1, c. $157-159$.

6. Watson G. S. Smooth regression analysis. - Sankhyā, Ser. A, 1964, v. 26, p. 359-372.

7. Hsing T. Estimating the parameters of rare events. - Stochastic Processes Appl., 1991 , v. 37, № 1, p. 117-139.

8. Smith R. L., Weissman I. Estimating the extremal index. - J. R. Statist. Soc. B, 1994 , v. 56, № 3, p. 515-528.

9. Deheuvels $P$. Conditions nécessaires et suffisantes de convergence ponctuelle presque sure et uniforme presque sure des estimateurs de la densité. - C. R. Acad. Sci. Paris, Ser. A, 1974, v. 278, p. 1217-1220.

10. Wolverton C. T., Wagner T. I. Asymptotically optimal discriminant functions for pattern classification. - IEEE Trans. Inform. Theory, 1969, v. 15, p. 258-266.

11. Paditz $L$. On the analytical structure of the constant in the nonuniform version of the Esseen inequality. - Statistics, 1989, v. 20, № 3, p. 453-464.

12. Жиганов И. С. Об уточнении верхней константы в остаточном члене ЦПТ. - В с6.: Проблемы устойчи вости стохастических моделей. М.: ВНИИСИ, 1982, с. 109115.

13. Collomb G. Quelques propriétés de la méthode du noyau pour l'estimation non parametrique de la régression en un point fixé. - C. R. Acad. Sci. Paris, Ser. A, 1977, v. 285, № 4 , p. $289-292$.

14. Devroye $L$. Necessary and sufficient conditions for the pointwise convergence of nearest neighbour regression function estimates. - Z . Wahrscheinlichkeitstheor. verw. Geb., 1982, B. 61, H. 4, S. 467-481.

15. Noda K. Estination of a regression function by Parzen kernel-type density estimators. - Ann. Inst. Statist. Math., 1976, v. 28, № 2, p. 221-234.

16. Parzen E. On estimation of a probability density function and mode. - Ann. Math. Statist., 1962, v. 33, № 3, p. 1065-1076.

17. Roussas G. G. Nonparametric regression estimation under mixing condition. - Stoch. Process. Appl., 1990, v. 36, № 1, p. 107-116.

18. Schuster E. F., Yakowitz S. Contributions to the theory of nonparametric regression with application to system identification. - Ann. Statist., 1979, v. 7, № 1, p. 139-149.

19. Rosenblatt $M$. Remarks on some nonparametric estimates of a density. - Ann. Math. Statist., 1956, v. 27, p. 832-835.

20. Алексеев $B$. $Г$. Некоторые вопросы статистического оценивания кривой регрессии. - Теория вероятн. и ее примен., 1991, т. 36, в. 2, с. 209-216.

21. Hill B. M. A simple general approach to inference about the tail of a distribution. Ann. Statist., 1975, v. 3, № 5, p. 1163-1174.

22. Haeusler E., Teugels $J$. L. On asymptotic normality of Hill's estimator for the exponent of regular variation. - Ann. Statist., 1985, v. 13, № 2, p. 743-756.

23. Csörgö S., Deheuvels $P$., Mason D. M. Kernel estimates of the tail index of the distribution. - Ann. Statist., 1985, v. 13, № 3, p. 1050-1077.

24. Csörgö M., Horváth L., Révész $P$. On the optimality of estimating the tail index and a naive estimator. - Austral. J. Math., 1987, v. 29, № 2, p. 166-178. 
25. Davis R., Resnick S. Tail estimates motivated by extreme value theory. - Ann. Statist., 1984, v. 12 , № 4, p. 1476-1487.

26. Smith R. L. Estimating tails of probability distributions. - Ann. Statist., 1987, v. 15, № 3, p. 1174-1207.

27. Bacro J. N., Brito $M$. Weak limiting behaviour of a simple tail Pareto-index estimator. - J. Statist. Plan. Infer., 1995, v. 45, p. 7-19.

28. Novak S. Yu. Inference about the Pareto-type distribution. - In: Trans. 11th Prague Conf. Inform. Theory Statist. Decision Func. Random Processes. Prague: Academia, 1992, v. B, p. 251-258.

29. Falk $M$. Extreme quantile estimation in $\delta$-neighbourhoods of generalized Pareto distribution. - Statist. Probab. Lett., 1994, v. 20, № 1, p. 9-21.

30. Teugels J. L. On Pareto-type distributions. - В сб.: Четвертая международная Вильнюсская конференция по теории вероятностей и математической статистике. т. IV. Вильнюс: Ин-т матем. и кибернет. АН Лит. ССР, 1985, с. 302-304.

31. Dekkers A. L. M., Einmahl J. H. J., de Haan L. A moment estimator for the index of an extreme-value distribution. - Ann. Statist., 1989, v. 17, № 4, p. 1833-1855.

32. Resnick S., Starica C. Smoothing the Hill estimator. - Technical Report, School of Operations Research and Industrial Eng., Cornell Univ., 1995.

33. Mason D. M. Laws of large numbers for sums of extreme values. - Anr. Probab., 1982 , v. 10, № 3, p. 754-764.

34. Deheuvels P., Haeusler E., Mason D. M. Almost sure convergence of the Hill estimator. - Math. Proc. Cambridge Philos. Soc., 1988, v, 104, № 2, p. 371-381.

35. Dekkers A. L. M., de Haan $L$. On the estimation of the extreme-value index and large quantile estimation. - Ann. Statist., 1989, v. 17, № 4, p. 1795-1832.

36. Hall $P$. On some simple estimates of an exponent of regular variation, - J. Roy. Statist. Soc. B, 1982 , v. 44, p. 37-42.

37. Hall P., Teugels J. L., Vanmarcke A. The abscissa of convergence of Laplace transforms. - J. Appl. Probab,, 1992, v. 29, № 2, p. 353-362.

38. Петров $B$. В. Предельные теоремы для сумм независимых случайных величик. M.: Наука, 1987, 320 c.

39. Seneta E. Regularly varying functions. (Lecture Notes Math. V. 508.) Berlin-Heidelberg: Springer-Verlag, 1976, $112 \mathrm{p}$.

40. Rice J., Rosenblatt $M$. Estimation of the log survivor function and hazard estimation. - Sankhyā, Ser. A, 1976, v. 38, № 1, p. 60-78.

41. Singpurwalla N. D., Wong M.-Y. Kernel estimators of the failure-rate function and density estimation: an analogy. - J. Amer. Statist. Assoc., 1983, v. 78, № 382, p. $478-481$.

42. Watson G. S., Leadbetter M. R. Hazard analysis. I. - Biometrika, 1964, v. 51, p. $175-184$.

43. Sethuraman J., Singpurwalla N. D. Large sample estimates and uniform confidence bounds for the failure-rate function based on a naive estimator. - Ann. Statist., 1981 , v. 9, № 3, p. 628-632. 